\title{
A MULTIPLICATIVE SCHWARZ METHOD AND ITS APPLICATION TO NONLINEAR ACOUSTIC-STRUCTURE INTERACTION*
}

\author{
Roland Ernst $^{1}$, Bernd Flemisch $^{2}$ And Barbara Wohlmuth ${ }^{1}$
}

\begin{abstract}
A new Schwarz method for nonlinear systems is presented, constituting the multiplicative variant of a straightforward additive scheme. Local convergence can be guaranteed under suitable assumptions. The scheme is applied to nonlinear acoustic-structure interaction problems. Numerical examples validate the theoretical results. Further improvements are discussed by means of introducing overlapping subdomains and employing an inexact strategy for the local solvers.
\end{abstract}

Mathematics Subject Classification. 74F10, 65B99, 65M12.

Received November 20, 2007. Revised August 14, 2008.

Published online April 8, 2009.

\section{INTRODUCTION}

We present a new Schwarz type domain decomposition method for nonlinear problems. Such methods typically lead to schemes where an outer iteration for the subproblem correction and an inner subspace iteration on each subproblem have to be applied $[15,17,18]$. Our method constitutes, by means of a Gauss-Seidel type outer iteration, a multiplicative variant of the straightforward additive scheme introduced in [7], and further improved in $[1,5]$.

In Section 1, we first recall the general setting and the theoretical results of the additive Schwarz method for nonlinear problems. Based on this framework, we introduce and analyze the corresponding multiplicative version. In the subsequent sections, the domain decomposition scheme is applied to nonlinear acoustic-structure interaction problems. In particular, we extend the elasto-acoustic problem formulation of [8] to geometrically nonlinear structures [6], and nonlinear acoustic wave propagation [13]. After introducing the single field problems in Section 2, we present the coupled problem formulation in Section 3, yielding two numerical schemes using different solvers for the subproblems, a Newton-like and a fixed point iteration scheme. We finally present the results of several numerical examples in Section 4. First, the theoretical results are validated and the convergence behavior of the additive and the multiplicative variant are compared. Moreover, further possible improvements of the scheme are discussed, one by the introduction of a small subregion where both subdomains overlap, and another by employing an inexact strategy for the local linear solver.

Keywords and phrases. Schwarz method, fluid-structure interaction, coupled problems, nonlinear elasticity, nonlinear acoustics, elasto-acoustic.

* This work was sponsored by the Deutsche Forschungsgemeinschaft, WO 671/6-1.

1 Institute of Applied Analysis and Numerical Simulation, University of Stuttgart, Germany.

2 Institute of Hydraulic Engineering, University of Stuttgart, Germany. bernd@iws.uni-stuttgart.de 


\section{Two SchWARZ METHOdS FOR NONLINEAR PROBLEMS}

The system we want to investigate is

$$
F\left(x^{*}\right):=\left(\begin{array}{c}
F_{\mathrm{e}}\left(x^{*}\right) \\
F_{\mathrm{a}}\left(x^{*}\right)
\end{array}\right)=0
$$

where $F_{\mathrm{e}}$ and $F_{\mathrm{a}}$ stand for the elastic and acoustic subproblems, respectively. We remark that this specific decomposition is purely motivated by the application presented afterwards, and that the theoretical results presented here in this section are general and do not depend on the physical origin of the subproblems. Moreover, our results can be extended to the case of more than two subproblems, and we will clearly indicate how this can be done. In the first part, we present the most straightforward alternative by means of a non-overlapping block Jacobi scheme and repeat the results provided by [7]. Second, we additionally introduce a multiplicative variant of the scheme yielding an improved convergence behavior. A major reason for the potential efficiency of our approach, even in the two-subdomain case, is that often only one part of the problem equations is nonlinear. Thus, one can exploit the structure of system (1.1) by constructing iterative schemes, where the nonlinear equations are decoupled from the linear ones in each iteration step.

\subsection{A nonlinearly preconditioned Richardson scheme}

We apply an additive scheme within the framework of nonlinear domain decomposition methods provided by [7], and borrow the notation and the ideas from [1,5]. Indicating with $m$ the global dimension of the system (1.1), we partition the set $\{1, \ldots, m\}$ into the index sets $I_{\mathrm{e}}$ and $I_{\mathrm{a}}$ corresponding to the structural and to the acoustic degrees of freedom, respectively, and set $m_{i}:=\left|I_{i}\right|, I_{i}:=\left\{i_{1}, \ldots, i_{m_{i}}\right\}, i=\mathrm{e}$, a. Defining the prolongation matrices $E_{i}^{\mathrm{T}} \in \mathbb{R}^{m \times m_{i}}$ and the restriction matrices $E_{i} \in \mathbb{R}^{m_{i} \times m}$ by

$$
\left(E_{i}\right)_{k \ell}:=\left\{\begin{array}{ll}
1, & \ell=i_{k}, \\
0, & \text { else }
\end{array} \quad i=\mathrm{e}, \mathrm{a}\right.
$$

we set $P_{i}:=E_{i}^{\mathrm{T}} E_{i} \in \mathbb{R}^{m \times m}$, and define the subspaces $V_{\mathrm{e}}$ and $V_{\mathrm{a}}$ by $V_{i}:=P_{i} \mathbb{R}^{m}$. For the different parts we have $F_{i}=P_{i} F$. Given $y \in \mathbb{R}^{m}$, the correction $T_{i}(y) \in V_{i}$ is defined as the solution of the subspace problem

$$
F_{i}\left(y-T_{i}(y)\right)=0, \quad i=\mathrm{e}, \mathrm{a}
$$

In general, each subspace problem is nonlinear and can be solved by, e.g., Newton's method. If for example the acoustic part consisted of a linear model, (1.2) would give the standard linear subspace correction

$$
T_{\mathrm{a}}(x)=E_{\mathrm{a}}^{\mathrm{T}}\left(K_{\mathrm{a}}^{*}\right)^{-1} E_{\mathrm{a}} F_{\mathrm{a}}(x),
$$

where $K_{\mathrm{a}}^{*}:=E_{\mathrm{a}} F^{\prime}(x) E_{\mathrm{a}}^{\mathrm{T}}$ would actually be independent of $x$, with $F^{\prime}(x)$ denoting the Jacobian of $F(x)$.

In the spirit of $[5]$, we introduce a new function

$$
\mathcal{F}(x):=T_{\mathrm{e}}(x)+T_{\mathrm{a}}(x)
$$

referred to as nonlinearly preconditioned $F(x)$, and attempt to solve

$$
\mathcal{F}\left(x^{*}\right)=0
$$

instead of (1.1). The solvability of the subproblems (1.2) and the equivalence of the two nonlinear systems (1.1) and (1.3) are stated in the following theorem which is proved in [5] using the results from [7]. 
Theorem $1.1([5,7])$. Let the following assumptions be satisfied:

(A1) The Jacobian $J(x):=F^{\prime}(x)$ exists and is continuous in a neighborhood $D$ of the exact solution $x^{*}$.

(A2) The matrix $J\left(x^{*}\right)$ is nonsingular.

(A3) The matrices $E_{i} J\left(x^{*}\right) E_{i}^{T}$ are nonsingular.

Then, the subproblems (1.2) are all uniquely solvable in a neighborhood of $x^{*}$ in D. Moreover, the nonlinear systems (1.1) and (1.3) are locally equivalent, i.e., they have the same solution in a neighborhood of $x^{*}$ in D.

It remains to solve the preconditioned system (1.3). As suggested by [7], we employ a standard Richardson iteration, namely, given $x^{(k)}$ and a damping parameter $\omega$, we calculate in each iteration step

$$
x^{(k+1)}:=\Phi\left(x^{(k)}\right), \text { with } \Phi(x):=x-\omega \mathcal{F}(x) .
$$

The expected convergence rate of (1.4) can be given in terms of the linearized problem $A x-b=0$ with $A:=J\left(x^{*}\right), b:=A x^{*}$. In this case, the subspace iteration (1.4) reduces to the well-known linear additive Schwarz method [18] with the iteration matrix

$$
M_{\omega}:=I-\omega \sum_{i=\mathrm{e}, \mathrm{a}} E_{i}^{\mathrm{T}} A_{i}^{-1} E_{i} A, \quad A_{i}:=E_{i} A E_{i}^{\mathrm{T}} .
$$

Assuming

$$
\left\|M_{\omega}\right\| \leq \zeta<1,
$$

the convergence behavior is described by the following theorem.

Theorem $1.2([7])$. Assume (A1)-(A4), and let $\zeta^{\prime} \in(\zeta, 1)$. Then there is a neighborhood $D\left(\zeta^{\prime}\right)$ of $x^{*}$ such that

$$
\|\Phi(y)-\Phi(z)\| \leq \zeta^{\prime}<1, \quad y, z \in D\left(\zeta^{\prime}\right),
$$

i.e., the nonlinear subspace iteration (1.4) converges in $D\left(\zeta^{\prime}\right)$ and has the same asymptotic convergence rate $\zeta$ as the linear iteration applied to the linearized equations.

Remark 1.3. One possible improvement is to replace the simple Richardson iteration in (1.4) by an inexact Newton method. This yields so-called ASPIN methods, i.e., Additive Schwarz Preconditioned Inexact Newton methods. They have been introduced in [5], and a proof of locally quadratic convergence under essentially the same assumptions (A1)-(A3) is provided in [1]. Here, we will present an alternative way.

\subsection{A multiplicative preconditioner}

In contrast to the nonlinearly preconditioned system (1.3), referred to as additive or Jacobi variant in the sequel, we can define a multiplicative or Gauss-Seidel variant by setting

$$
\mathcal{F}_{\mathrm{m}}(x):=T_{\mathrm{e}}(x)+T_{\mathrm{a}}\left(x-T_{\mathrm{e}}(x)\right) .
$$

Similar to (1.3), we now try to solve the nonlinearly preconditioned system

$$
\mathcal{F}_{\mathrm{m}}\left(x^{*}\right)=0 .
$$

To the best knowledge of the authors, no theory for this multiplicative type of nonlinear subspace preconditioning exists. In order to analyze this case, we first need the following lemma.

Lemma 1.4. Under Assumptions (A1)-(A3), the Jacobian $\mathcal{J}(x):=\mathcal{F}_{m}^{\prime}(x)$ is nonsingular in a neighborhood of $x^{*}$. 
Proof. Setting $\bar{x}:=x-T_{\mathrm{e}}(x)$ and applying the chain rule, we have that

$$
\mathcal{J}(x)=I-\left(I-T_{\mathrm{a}}^{\prime}(\bar{x})\right)\left(I-T_{\mathrm{e}}^{\prime}(x)\right) .
$$

We follow the argumentation for the additive case from $[1,5]$. There, it is shown that

$$
T_{i}^{\prime}(x)=J_{i}^{-1}\left(x-T_{i}(x)\right) J\left(x-T_{i}(x)\right),
$$

where $J_{i}^{-1}\left(x-T_{i}(x)\right)$ is understood as the subdomain inverse, i.e., $J_{i}^{-1}\left(x-T_{i}(x)\right):=E_{i}^{\mathrm{T}}\left(E_{i} J_{i}\left(x-T_{i}(x)\right) E_{i}^{\mathrm{T}}\right)^{-1} E_{i}$, which is known to exist by Assumption (A3). By assumption, all terms are continuous and, thus, $J\left(x-T_{i}(x)\right)$ and $J\left(\bar{x}-T_{i}(\bar{x})\right)$ are close to $J(x)$ in a neighborhood of $x^{*}$. Therefore, $\mathcal{J}(x)$ is also continuous around $x^{*}$.

In order to show the regularity of $\mathcal{J}(x)$, we introduce the matrix

$$
B(x):=I-\left(I-J_{\mathrm{a}}^{-1}(x) J(x)\right)\left(I-J_{\mathrm{e}}^{-1}(x) J(x)\right) .
$$

Again by continuity arguments, we have that $B(x)$ is continuous in a neighborhood of $x^{*}$, and that

$$
\lim _{x \rightarrow x^{*}} \mathcal{J}(x)=\lim _{x \rightarrow x^{*}} B(x)=\mathcal{J}\left(x^{*}\right) .
$$

In the special case of a decomposition into two nonoverlapping sets, it is easy to explicitly construct the inverse $B^{-1}(x)$ in terms of the subdomain Jacobians. In particular, if we omit the dependence on $x$ for the moment, it is easy to see that

$$
B=\left(\begin{array}{cc}
J_{\mathrm{e}}^{-1} & 0 \\
J_{\mathrm{a}}^{-1} J_{\mathrm{ae}} J_{\mathrm{e}}^{-1} & J_{\mathrm{a}}^{-1}
\end{array}\right) J,
$$

and, therefore,

$$
B^{-1}=J^{-1}\left(\begin{array}{cc}
J_{\mathrm{e}} & 0 \\
-J_{\mathrm{ae}} & J_{\mathrm{a}}
\end{array}\right) .
$$

Using the regularity of $B(x)$, the equality (1.7), and continuity, we obtain the desired regularity of $\mathcal{J}(x)$ close to $x^{*}$.

Remark 1.5. The generalization of the proof given above to the case of many nonoverlapping subsets is straightforward. However, in the case of overlap, the proof of the regularity of $B(x)$ remains to be given.

Concerning the equivalence of (1.1) and (1.6), we show the following result in analogy to Theorem 1.1.

Theorem 1.6. Under Assumptions (A1)-(A3) from Theorem 1.1, the nonlinear systems (1.1) and (1.6) are equivalent in the sense that they have the same solution in a neighborhood of $x^{*}$ in D.

Proof. We follow the lines of the proof of Theorem 1.1 provided in [5]. Assuming that $x^{*}$ solves (1.1), we have $F_{i}\left(x^{*}\right)=0, i=\mathrm{e}$, a. Comparing this with the definition of $T_{i}$ in (1.2), and using the local unique solvability guaranteed by Theorem 1.1, we have $T_{i}\left(x^{*}\right)=0$. Therefore, $x^{*}$ is also a solution of (1.6). It remains to show that $x^{*}$ is unique in a local neighborhood. By Lemma 1.4, the Jacobian $\mathcal{F}_{\mathrm{m}}^{\prime}\left(x^{*}\right)$ is nonsingular, and therefore, according to the inverse function theorem, the solution is locally unique.

In order to solve (1.6), we apply the damped Richardson iteration (1.4) with $\mathcal{F}$ replaced by $\mathcal{F}_{\mathrm{m}}$, namely,

$$
x^{(k+1)}:=\Phi_{\mathrm{m}}\left(x^{(k)}\right), \text { with } \Phi_{\mathrm{m}}(x):=x-\omega \mathcal{F}_{\mathrm{m}}(x) .
$$

We expect that an analogue of Theorem 1.2 has to hold, i.e., the convergence rate should be close to the one of the corresponding linear iteration applied to the linearized problem. In particular, the Richardson iteration 
in the linear case described above reduces to the standard multiplicative Schwarz method with the iteration matrix

$$
M_{\omega, \mathrm{m}}:=I-\omega\left(I-\prod_{i=\mathrm{e}, \mathrm{a}}\left(I-E_{i}^{\mathrm{T}} A_{i}^{-1} E_{i} A\right)\right) .
$$

Analogous to (A4), we now assume that

$$
\left\|M_{\omega, \mathrm{m}}\right\| \leq \zeta<1
$$

Theorem 1.7. Assume (A1)-(A3), $\left(\mathrm{A}^{\prime}\right)$, and let $\zeta^{\prime} \in(\zeta, 1)$. Then there is a neighborhood $D\left(\zeta^{\prime}\right)$ of $x^{*}$ such that

$$
\left\|\Phi_{m}(y)-\Phi_{m}(z)\right\| \leq \zeta^{\prime}<1, \quad y, z \in D\left(\zeta^{\prime}\right) .
$$

i.e., the nonlinear subspace iteration (1.8) converges in $D\left(\zeta^{\prime}\right)$ and has the same asymptotic convergence rate $\zeta$ as the linear iteration applied to the linearized equations.

Proof. We alternate the proof of Theorem 1.2 presented in [7]. There, the analysis is based on weaker assumptions employing a general difference quotient $D F$. Since (A1) is satisfied, we can choose $D F\left(x^{\prime}, x^{\prime \prime}\right) \in \mathbb{R}^{m \times m}$ as

In [7], it is shown that

$$
D F\left(x^{\prime}, x^{\prime \prime}\right):=\int_{0}^{1} J\left(x^{\prime}+t\left(x^{\prime \prime}-x^{\prime}\right)\right) \mathrm{d} t .
$$

$$
T_{i}\left(x^{\prime}\right)-T_{i}\left(x^{\prime \prime}\right)=D T_{i}\left(x^{\prime}, x^{\prime \prime}\right)\left(x^{\prime}-x^{\prime \prime}\right),
$$

for all $x^{\prime}, x^{\prime \prime}$ sufficiently close to $x^{*}$, where $D T_{i}\left(x^{\prime}, x^{\prime \prime}\right)$ is defined as

$$
D T_{i}\left(x^{\prime}, x^{\prime \prime}\right):=E_{i}^{\mathrm{T}}\left(E_{i} D F E_{i}^{\mathrm{T}}\right)^{-1} E_{i} D F, \quad D F:=D F\left(x^{\prime}-T_{i}\left(x^{\prime}\right), x^{\prime \prime}-T_{i}\left(x^{\prime \prime}\right)\right) .
$$

Moreover, $D T_{i}\left(x^{\prime}, x^{\prime \prime}\right)$ satisfies

$$
D T_{i}\left(x^{\prime}, x^{\prime \prime}\right) \rightarrow J_{i}^{-1}\left(x^{*}\right) J\left(x^{*}\right) \quad \text { as } x^{\prime}, x^{\prime \prime} \rightarrow x^{*}
$$

In view of $(1.5)$, and starting from $x^{\prime}, x^{\prime \prime}$, we set $\bar{x}:=x^{\prime}-T_{\mathrm{e}}\left(x^{\prime}\right), \widehat{x}:=x^{\prime \prime}-T_{\mathrm{e}}\left(x^{\prime \prime}\right)$, and derive from (1.9) the relation

$$
\begin{aligned}
T_{\mathrm{a}}(\bar{x})-T_{\mathrm{a}}(\widehat{x}) & =D T_{\mathrm{a}}(\bar{x}, \widehat{x})(\bar{x}-\widehat{x}) \\
& =D T_{\mathrm{a}}(\bar{x}, \widehat{x})\left(x^{\prime}-x^{\prime \prime}-\left(T_{\mathrm{e}}\left(x^{\prime}\right)-T_{\mathrm{e}}\left(x^{\prime \prime}\right)\right)\right) \\
& =D T_{\mathrm{a}}(\bar{x}, \widehat{x})\left(I-D T_{\mathrm{e}}\left(x^{\prime}, x^{\prime \prime}\right)\right)\left(x^{\prime}-x^{\prime \prime}\right) .
\end{aligned}
$$

The definition $(1.5)$ of $\mathcal{F}_{\mathrm{m}}$ yields

$$
\Phi_{\mathrm{m}}\left(x^{\prime}\right)-\Phi_{\mathrm{m}}\left(x^{\prime \prime}\right)=\left(x^{\prime}-x^{\prime \prime}\right)-\omega\left(\left(T_{\mathrm{e}}\left(x^{\prime}\right)-T_{\mathrm{e}}\left(x^{\prime \prime}\right)\right)+\left(T_{\mathrm{a}}(\bar{x})-T_{\mathrm{a}}(\widehat{x})\right)\right),
$$

and inserting (1.9) and (1.11), we have

$$
\Phi_{\mathrm{m}}\left(x^{\prime}\right)-\Phi_{\mathrm{m}}\left(x^{\prime \prime}\right)=\left(I-\omega\left(I-\left(I-D T_{\mathrm{a}}(\bar{x}, \widehat{x})\right)\left(I-D T_{\mathrm{e}}\left(x^{\prime}, x^{\prime \prime}\right)\right)\right)\right)\left(x^{\prime}-x^{\prime \prime}\right) .
$$

Employing (1.10) and the fact that $\bar{x}, \widehat{x} \rightarrow x^{*}$ as $x^{\prime}, x^{\prime \prime} \rightarrow x^{*}$, we see that

$$
I-\omega\left(I-\left(I-D T_{\mathrm{a}}(\bar{x}, \widehat{x})\right)\left(I-D T_{\mathrm{e}}\left(x^{\prime}, x^{\prime \prime}\right)\right)\right) \rightarrow M_{\omega, \mathrm{m}} \quad \text { as } x^{\prime}, x^{\prime \prime} \rightarrow x^{*} .
$$

Therefore, (A4') implies the desired assertion.

Remark 1.8. By using induction arguments, the proof of Theorem 1.7 can easily be generalized to the case of many subdomains.

Remark 1.9. As mentioned in Remark 1.3, a further improvement could be achieved by employing an inexact Newton method instead of the Richardson scheme (1.8), yielding a multiplicative variant of ASPIN methods [1,5]. 


\section{Model Equations}

In the following two application-oriented sections of this paper we derive the different nonlinear subproblems of system (1.1) in order to apply the theoretical results. The field of fluid-structure interactions contains many applications for elasto-acoustic coupling problems, especially nonlinear ones. Linear elasticity is restricted to the case of small deformations. Nonlinear acoustic effects including dissipation and generation of higher harmonics play an important role in ultrasonics. The choice of a velocity potential or pressure based formulation for the acoustic part and of a displacement one for the elastic part results in Dirichlet-Neumann type interface conditions. We start with introducing the single field problems of elasto-dynamics and of acoustics, providing the strong and weak continuous formulations.

\subsection{Nonlinear structural dynamics}

We investigate the deformation of an elastic body $\Omega^{\mathrm{e}} \subset \mathbb{R}^{d}$ with density $\rho_{\mathrm{e}}: \Omega^{\mathrm{e}} \rightarrow \mathbb{R}$ under given time dependent volume and surface forces $\boldsymbol{f}$ and $\boldsymbol{g}$. The boundary of $\Omega^{\mathrm{e}}$ with unit normal vector $\boldsymbol{n}_{\mathrm{e}}$ is the union of disjoint subsets $\Gamma_{\mathrm{N}}^{\mathrm{e}}$ and $\Gamma_{\mathrm{D}}^{\mathrm{e}}$, on which natural and essential data are given, respectively. If the strains remain small but the deformations become large, one has at least to consider the geometrically nonlinear elasticity setting. This amounts to using the full Green-St. Venant tensor

$$
E:=\frac{1}{2}\left(F^{\mathrm{T}} F-\mathrm{Id}\right)=\frac{1}{2}(C-\mathrm{Id}),
$$

with $F:=\mathrm{Id}+\operatorname{grad} \boldsymbol{u}$ the deformation gradient and $C:=F^{\mathrm{T}} F$ the right Cauchy-Green strain tensor. We have the constitutive law

$$
S=\lambda_{\mathrm{L}}(\operatorname{tr} E) \operatorname{Id}+2 \mu_{\mathrm{L}} E=\mathcal{C} E,
$$

defining the second Piola-Kirchhoff stress tensor $S$, with $\mathcal{C}$ the Hooke-tensor and the Lamé constants $\lambda_{\mathrm{L}}, \mu_{\mathrm{L}}$. The strong formulation for elasto-dynamic problems then reads as follows:

Given $\boldsymbol{u}_{0}, \boldsymbol{u}_{1}, \boldsymbol{f}: \Omega^{\mathrm{e}} \rightarrow \mathbb{R}^{d}$, find $\boldsymbol{u}: \Omega^{\mathrm{e}} \times(0, T) \rightarrow \mathbb{R}^{d}$ such that

$$
\rho_{\mathrm{e}} \ddot{\boldsymbol{u}}-\operatorname{div}(F S)=\boldsymbol{f} \quad \text { in } \Omega^{\mathrm{e}} \times(0, T),
$$

complemented by the boundary conditions

$$
\boldsymbol{u}=\mathbf{0} \text { on } \Gamma_{\mathrm{D}}^{\mathrm{e}} \times(0, T), \quad F S \boldsymbol{n}_{\mathrm{e}}=\boldsymbol{g} \text { on } \Gamma_{\mathrm{N}}^{\mathrm{e}} \times(0, T),
$$

and initial conditions

$$
\boldsymbol{u}(\cdot, 0)=\boldsymbol{u}_{0}, \dot{\boldsymbol{u}}(\cdot, 0)=\boldsymbol{u}_{1} \text { in } \Omega^{\mathrm{e}} .
$$

Under additional assumptions on the data, it is possible to establish the well-posedness of $(2.1)-(2.3 c), c f .[6,16]$. For the weak formulation, it is natural to use the space

$$
X^{\mathrm{e}}:=\left(H_{\Gamma_{\mathrm{D}}^{\mathrm{e}}}^{1}\left(\Omega^{\mathrm{e}}\right)\right)^{d}:=\left\{\boldsymbol{v} \in\left(H^{1}\left(\Omega^{\mathrm{e}}\right)\right)^{d}: \boldsymbol{v}=0 \text { on } \Gamma_{\mathrm{D}}^{\mathrm{e}}\right\} .
$$

We employ the standard setting of Sobolev spaces for evolution problems; the space $L^{2}(0, T ; X)$ consists of functions $t \mapsto v(t)$ which are Lebesgue-measurable on $(0, T)$ with respect to the measure $\mathrm{d} t$, such that

$$
\|v\|_{L^{2}(0, T ; X)}:=\left(\int_{0}^{T}\|v(t)\|_{X}^{2} \mathrm{~d} t\right)^{1 / 2}<\infty .
$$

The resulting variational problem reads:

Find $\boldsymbol{u} \in L^{2}\left(0, T ; X^{\mathrm{e}}\right), \dot{\boldsymbol{u}} \in L^{2}\left(0, T ; L^{2}\left(\Omega^{\mathrm{e}}\right)\right)$, and $\ddot{\boldsymbol{u}} \in L^{2}\left(0, T ; H^{-1}\left(\Omega^{\mathrm{e}}\right)\right)$ such that for all times $t \in(0, T)$

$$
\left\langle\rho_{\mathrm{e}} \ddot{\boldsymbol{u}}, \boldsymbol{v}\right\rangle_{\Omega^{\mathrm{e}}}+a_{\mathrm{e}}(\boldsymbol{u}, \boldsymbol{v})=l_{\mathrm{e}}(\boldsymbol{v}):=(\boldsymbol{f}, \boldsymbol{v})_{\Omega^{\mathrm{e}}}+(\boldsymbol{g}, \boldsymbol{v})_{\Gamma_{\mathrm{N}}^{\mathrm{e}}}, \quad \boldsymbol{v} \in X^{\mathrm{e}},
$$


together with initial conditions (2.3c). In (2.4), $\langle\cdot, \cdot\rangle_{\Omega^{\mathrm{e}}}$ indicates the duality product on $X^{\mathrm{e}} \times\left(X^{\mathrm{e}}\right)^{\prime}$. Transforming to the weak setting, the nonlinearity in $(2.1)$ results in the fact that $a_{\mathrm{e}}(\cdot, \cdot)$ is nonlinear in the first and linear in the second argument, i.e., $a_{\mathrm{e}}(\boldsymbol{u}, \boldsymbol{v}):=\sum_{i=1}^{4} a_{\mathrm{e}, i}(\boldsymbol{u}, \boldsymbol{v})$, is given by, cf. [6],

$$
\begin{aligned}
a_{\mathrm{e}, 1}(\boldsymbol{u}, \boldsymbol{v}) & :=(\mathcal{C} \varepsilon(\boldsymbol{u}), \varepsilon(\boldsymbol{v}))_{\Omega^{\mathrm{e}}}, & a_{\mathrm{e}, 2}(\boldsymbol{u}, \boldsymbol{v}) & :=\frac{1}{2}\left(\mathcal{C}\left[(\operatorname{grad} \boldsymbol{u})^{\mathrm{T}} \operatorname{grad} \boldsymbol{u}\right], \operatorname{grad} \boldsymbol{v}\right)_{\Omega^{\mathrm{e}}}, \\
a_{\mathrm{e}, 3}(\boldsymbol{u}, \boldsymbol{v}) & :=(\operatorname{grad} \boldsymbol{u C} \varepsilon(\boldsymbol{u}), \operatorname{grad} \boldsymbol{v})_{\Omega^{\mathrm{e}}}, & a_{\mathrm{e}, 4}(\boldsymbol{u}, \boldsymbol{v}) & :=\frac{1}{2}\left(\operatorname{grad} \boldsymbol{u C}\left[(\operatorname{grad} \boldsymbol{u})^{\mathrm{T}} \operatorname{grad} \boldsymbol{u}\right], \operatorname{grad} \boldsymbol{v}\right)_{\Omega^{\mathrm{e}},},
\end{aligned}
$$

with the linearized strain tensor

$$
\varepsilon(\boldsymbol{u}):=\frac{1}{2}\left(\operatorname{grad} \boldsymbol{u}+[\operatorname{grad} \boldsymbol{u}]^{\mathrm{T}}\right) .
$$

\subsection{Linear structure dynamics}

Assuming small deformations and small strain, it is sufficient to investigate the linear system

$$
\rho_{\mathrm{e}} \ddot{\boldsymbol{u}}-\operatorname{div}(\sigma)=\boldsymbol{f} \quad \text { in } \Omega^{\mathrm{e}} \times(0, T),
$$

with the stress tensor $\sigma$ given by Hooke's Law

$$
\sigma=\lambda_{\mathrm{L}}(\operatorname{tr} \varepsilon) \operatorname{Id}+2 \mu_{\mathrm{L}} \varepsilon,
$$

complemented by the boundary conditions

$$
\boldsymbol{u}=\mathbf{0} \text { on } \Gamma_{\mathrm{D}}^{\mathrm{e}} \times(0, T), \quad \sigma \boldsymbol{n}_{\mathrm{e}}=\boldsymbol{g} \text { on } \Gamma_{\mathrm{N}}^{\mathrm{e}} \times(0, T),
$$

and initial conditions (2.3c). The weak form is analogous to $(2.4)$ with $a_{\mathrm{e}}(\cdot, \cdot)$ replaced by $a_{\mathrm{e}}^{\operatorname{lin}}(\cdot, \cdot):=a_{\mathrm{e}, 1}(\cdot, \cdot)$.

\subsection{Nonlinear acoustic waves}

For the description of the acoustic wave propagation within a domain $\Omega^{\mathrm{a}} \subset \mathbb{R}^{d}$, we use the wave equation for the velocity potential $\psi$, i.e., $\boldsymbol{v}_{\mathrm{a}}=-\operatorname{grad} \psi$ with $\boldsymbol{v}_{\mathrm{a}}$ denoting the acoustic velocity field. The acoustic pressure $p_{\mathrm{a}}$ is then related to the acoustic velocity potential $\psi$ by

$$
p_{\mathrm{a}}=\rho_{\mathrm{a}} \dot{\psi}
$$

with $\rho_{\mathrm{a}}: \Omega^{\mathrm{a}} \rightarrow \mathbb{R}$ the mean density of the fluid. This connection will be important for an interface condition of force transition in the coupled system. Analogous to before, $\boldsymbol{n}_{\mathrm{a}}$ denotes the unit normal vector to the boundary of $\Omega^{\mathrm{a}}$, which is the union of disjoint subsets $\Gamma_{\mathrm{N}}^{\mathrm{a}}$ and $\Gamma_{\mathrm{D}}^{\mathrm{a}}$. Indicating with $c: \Omega^{\mathrm{a}} \rightarrow \mathbb{R}$ the speed of sound, the acoustic problem with the nonlinear wave equation derived by Kuznetsov reads as follows $[11,13]$ :

Given $\psi_{0}, \psi_{1}: \Omega^{\mathrm{a}} \rightarrow \mathbb{R}$, find $\psi: \Omega^{\mathrm{a}} \times(0, T) \rightarrow \mathbb{R}$ such that

$$
\frac{1}{c^{2}} \ddot{\psi}-\operatorname{div} \operatorname{grad} \psi=\frac{1}{c^{2}} \frac{\partial}{\partial t}\left(b \operatorname{div} \operatorname{grad} \psi+\frac{1}{c^{2}} \frac{B}{2 A} \dot{\psi}^{2}+(\operatorname{grad} \psi)^{2}\right) \quad \text { in } \Omega^{\mathrm{a}} \times(0, T),
$$

with boundary conditions

$$
\psi=0 \text { on } \Gamma_{\mathrm{D}}^{\mathrm{a}} \times(0, T), \quad \operatorname{grad} \psi \cdot \boldsymbol{n}_{\mathrm{a}}=g \text { on } \Gamma_{\mathrm{N}}^{\mathrm{a}} \times(0, T),
$$

and initial conditions

$$
\psi(\cdot, 0)=\psi_{0}, \dot{\psi}(\cdot, 0)=\psi_{1} \quad \text { in } \Omega^{\mathrm{a}} .
$$


The parameter $b$ involves thermodynamical effects as well as the viscosity of the fluid, whereas $B / A$ is called the nonlinearity parameter of the fluid. From a modeling point of view one could extend (2.5) to a higher accuracy relation $p_{\mathrm{a}}=\rho_{\mathrm{a}}\left(\dot{\psi}+\frac{1}{2}(\operatorname{grad} \psi)^{2}-\frac{1}{2 c^{2}} \dot{\psi}^{2}\right)$ [9], which would lead to a different force interface condition. But the occurrence of the nonlinear effects depends only on the nonlinear wave equation (2.6a). Due to this fact we consider here condition (2.5). Setting $X^{\mathrm{a}}:=H_{\Gamma_{\mathrm{D}}^{\mathrm{a}}}^{1}\left(\Omega^{\mathrm{a}}\right)$, the weak form of (2.6) reads:

Find $\psi \in L^{2}\left(0, T ; X^{\mathrm{a}}\right), \dot{\psi} \in L^{2}\left(0, T ; L^{2}\left(\Omega^{\mathrm{a}}\right)\right)$, and $\ddot{\psi} \in L^{2}\left(0, T ; H^{-1}\left(\Omega^{\mathrm{a}}\right)\right)$ such that for all times $t \in(0, T)$

$$
\left\langle\frac{1}{c^{2}} \ddot{\psi}, w\right\rangle_{\Omega^{\mathrm{a}}}+a_{\mathrm{a}}(\psi, w)=l_{\mathrm{a}}(w), \quad w \in X^{\mathrm{a}},
$$

in addition with the initial conditions $(2.6 \mathrm{c})$, where $a_{\mathrm{a}}(\psi, w):=a_{\mathrm{a}, 1}(\psi, w)+a_{\mathrm{a}, 2}(\psi, w)$ is splitted into one part deriving from the linear wave operator and another, which arrives from the nonlinear aspect

$$
\begin{aligned}
& a_{\mathrm{a}, 1}(\psi, w):=(\operatorname{grad} \psi, \operatorname{grad} w)_{\Omega^{\mathrm{a}}}, \\
& a_{\mathrm{a}, 2}(\psi, w):=\left(\frac{b}{c^{2}} \operatorname{grad} \dot{\psi}, \operatorname{grad} w\right)_{\Omega^{\mathrm{a}}}-\left\langle\frac{B / A}{c^{4}} \ddot{\psi}, \dot{\psi} w\right\rangle_{\Omega^{\mathrm{a}}}-\left(\frac{2}{c^{2}} \operatorname{grad} \dot{\psi}, \operatorname{grad} \psi w\right)_{\Omega^{\mathrm{a}}} .
\end{aligned}
$$

The linear functional is similarly defined as

$$
\begin{aligned}
l_{\mathrm{a}}(w) & :=l_{\mathrm{a}, 1}(w)+l_{\mathrm{a}, 2}(w) \\
& :=\langle g, w\rangle_{\Gamma_{\mathrm{N}}^{\mathrm{a}}}+\langle\dot{g}, w\rangle_{\Gamma_{\mathrm{N}}^{\mathrm{a}}} .
\end{aligned}
$$

\subsection{Linear acoustic waves}

The Kuznetsov equation (2.6a) easily allows a connection to the linear wave equation

$$
\frac{1}{c^{2}} \ddot{\psi}-\operatorname{div} \operatorname{grad} \psi=0 \quad \text { in } \Omega^{\mathrm{a}} \times(0, T),
$$

by setting the right hand side to zero. Hence, we have in $(2.7)$ the bilinear form $a_{\mathrm{a}}^{\operatorname{lin}}(\cdot, \cdot):=a_{\mathrm{a}, 1}(\cdot, \cdot)$ and the right hand side $l_{\mathrm{a}}^{\operatorname{lin}}(\cdot):=l_{\mathrm{a}, 1}(\cdot)$.

\section{Coupled systems}

In the following, the coupled problem of elasto-acoustics is presented. We first focus on the interface conditions, where the time derivative of the Dirichlet data from one side is coupled with the Neumann data from the other side. After that, we observe how these conditions enter into the variational formulation. Subsequent to the continuous weak formulation, the semi-discrete problem obtained after spatial discretization is presented.

\subsection{Structure-acoustic interface}

At a solid-fluid interface $\Gamma$, as depicted in Figure 1a, the continuity requires that the normal component of the mechanical surface velocity of the solid must coincide with the normal component of the acoustic velocity of the fluid. Thus, the following relation between the velocity $\boldsymbol{v}_{\mathrm{e}}$ of the solid expressed by the mechanical displacement $\boldsymbol{u}$ and the acoustic particle velocity $\boldsymbol{v}_{\text {a }}$ expressed by the acoustic scalar potential $\psi$ arises:

$$
0=\left(\boldsymbol{v}_{\mathrm{e}}-\boldsymbol{v}_{\mathrm{a}}\right) \cdot \boldsymbol{n}=\dot{\boldsymbol{u}} \cdot \boldsymbol{n}+\operatorname{grad} \psi \cdot \boldsymbol{n} \quad \text { on } \Gamma \times(0, T),
$$

where the unit normal vector field $\boldsymbol{n}$ on $\Gamma$ points outward of $\Omega^{\mathrm{e}}$. In addition, one has to consider the fact that the ambient fluid causes a surface force $\boldsymbol{f}_{\Gamma}=-p_{\mathrm{a}} \boldsymbol{n}=-\rho_{\mathrm{a}} \dot{\psi} \boldsymbol{n}$, see (2.5), which acts like a pressure load 
(a)

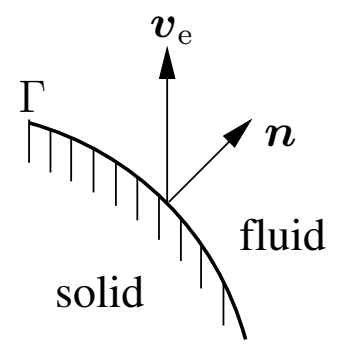

(b)

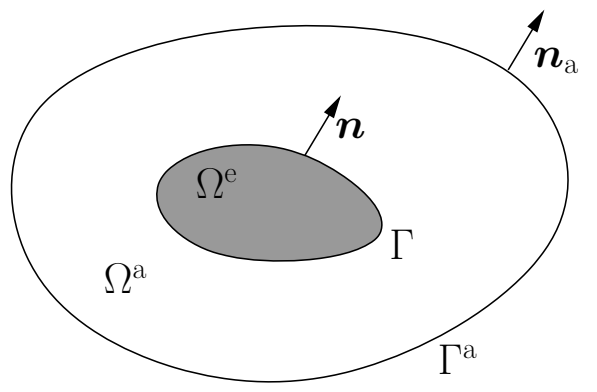

Figure 1. (a) Solid-fluid interface, (b) setup of the coupled elasto-acoustic problem.

on the solid. Therefore, a second coupling condition is given by

$$
F S \boldsymbol{n}+\rho_{\mathrm{a}} \dot{\psi} \boldsymbol{n}=\mathbf{0} \quad \text { on } \Gamma \times(0, T)
$$

For the acoustic part, we will apply the nonlinear wave equation according to Kutznetsov, which models the dynamics in a viscous heat-conducting fluid [13]. However, assuming motion of a potential character, the equations reduce to a single nonlinear wave equation [11]. Therewith, our formulation just supports longitudinal waves (neglecting shear waves) within the acoustic domain, and thus we just have to apply the continuity for the normal components of fluid particle velocity and mechanical velocity along the common interface.

\subsection{Coupled field formulation}

Let us consider a setup of a coupled mechanical-acoustic problem as shown in Figure 1b, where at the interface $\Gamma$ we have to consider the solid-fluid coupling. Now, within the domain $\Omega^{\mathrm{e}}$ the initial boundary value problem (2.3) for the mechanical field, within the domain $\Omega^{\mathrm{a}}$ the initial boundary value problem (2.6) for the acoustic field, and along the interface $\Gamma$ the coupling conditions according to (3.1) and (3.2) have to be satisfied. Transforming to the weak form, assuming that homogeneous Neumann data is given on $\Gamma^{\mathrm{a}}=\partial \Omega^{\mathrm{a}} \backslash \Gamma$, we obtain for the mechanical system

$$
\left\langle\rho_{\mathrm{e}} \ddot{\boldsymbol{u}}, \boldsymbol{v}\right\rangle_{\Omega^{\mathrm{e}}}+a_{\mathrm{e}}(\boldsymbol{u}, \boldsymbol{v})-\langle\boldsymbol{v}, F S \boldsymbol{n}\rangle_{\Gamma}=l_{\mathrm{e}}(\boldsymbol{v})
$$

and for the acoustic system

$$
\left\langle\frac{1}{c^{2}} \ddot{\psi}, w\right\rangle_{\Omega^{a}}+a_{\mathrm{a}}(\psi, w)+\langle w, \operatorname{grad} \psi \cdot \boldsymbol{n}\rangle_{\Gamma}+\langle w, \frac{b}{c^{2}} \overbrace{\operatorname{\gamma rad} \psi \cdot \boldsymbol{n}}\rangle_{\Gamma}=0,
$$

where $\gamma$ denotes the usual trace operator onto the solid-fluid interface $\Gamma$. It should be noted that the plus sign in (3.4) in front of the first boundary integral over $\Gamma$ is due to the choice of $\boldsymbol{n}$ to point outward with respect to the structure $\Omega^{\mathrm{e}}$, see Figure 1b. Incorporating the coupling conditions (3.1) and (3.2), we arrive at the following coupled system of equations

$$
\begin{aligned}
& \left\langle\rho_{\mathrm{e}} \ddot{\boldsymbol{u}}, \boldsymbol{v}\right\rangle_{\Omega^{\mathrm{e}}}+a_{\mathrm{e}}(\boldsymbol{u}, \boldsymbol{v})+\langle\rho_{\mathrm{a}} \boldsymbol{v} \cdot \boldsymbol{n}, \overbrace{\gamma \psi}^{\dot{\gamma}}\rangle_{\Gamma}=l_{\mathrm{e}}(\boldsymbol{v}), \\
& \left\langle\frac{1}{c^{2}} \ddot{\psi}, w\right\rangle_{\Omega^{a}}+a_{\mathrm{a}}(\psi, w)-\langle w, \overbrace{\gamma \boldsymbol{u} \cdot \boldsymbol{n}}^{\cdot}\rangle_{\Gamma}-\langle w, \frac{b}{c^{2}} \overbrace{\boldsymbol{\gamma} \cdot \boldsymbol{n}}^{\ddot{n}}\rangle_{\Gamma}=0,
\end{aligned}
$$

where the lower right hand side is zero due to the choice of homogeneous Neumann boundary conditions on $\Gamma^{\mathrm{a}}$. Before we perform a domain discretization, we multiply (3.6) by $\rho_{\mathrm{a}}$ in order to obtain a boundary integral similar to the one in (3.5). Thus, the matrices, occurring from a finite element discretization of these two boundary 
integrals, will be the negative transpose of each other. We finally arrive at the variational problem of finding $(\boldsymbol{u}, \psi) \in L^{2}\left(0, T ; X^{\mathrm{e}} \times X^{\mathrm{a}}\right)$ such that for all times $t \in(0, T)$ :

$$
\begin{aligned}
&\left\langle\rho_{\mathrm{e}} \ddot{\boldsymbol{u}}, \boldsymbol{v}\right\rangle_{\Omega^{\mathrm{e}}}+a_{\mathrm{e}}(\boldsymbol{u}, \boldsymbol{v})+\langle\rho_{\mathrm{a}} \boldsymbol{v} \cdot \boldsymbol{n}, \overbrace{\gamma \psi}^{\dot{\gamma \psi}}\rangle_{\Gamma}=l_{\mathrm{e}}(\boldsymbol{v}), \quad \boldsymbol{v} \in X^{\mathrm{e}}, \\
&\left\langle\frac{\rho_{\mathrm{a}}}{c^{2}} \ddot{\psi}, w\right\rangle_{\Omega^{a}}+a_{\mathrm{a}}\left(\psi, \rho_{\mathrm{a}} w\right)-\langle\rho_{\mathrm{a}} w, \overbrace{\boldsymbol{\gamma} \cdot \boldsymbol{n}}\rangle_{\Gamma}-\langle\rho_{\mathrm{a}} w, \frac{b}{c^{2}} \overbrace{\boldsymbol{\gamma} \cdot \boldsymbol{n}}^{*}\rangle_{\Gamma}=0, \quad w \in X^{\mathrm{a}} .
\end{aligned}
$$

Our main focus in this paper concentrates on the nonlinear elastic/linear acoustic system on the one hand and on the linear elastic/nonlinear acoustic system on the other. The problem formulations do not differ essentially from (3.7) because nearly all of the nonlinear effects are hidden in the definition of $a_{\mathrm{e}}(\cdot, \cdot)$ and $a_{\mathrm{a}}(\cdot, \cdot)$.

Nonlinear elastic/linear acoustic continuous system

Find $(\boldsymbol{u}, \psi) \in L^{2}\left(0, T ; X^{\mathrm{e}} \times X^{\mathrm{a}}\right),(\dot{\boldsymbol{u}}, \dot{\psi}) \in L^{2}\left(0, T ; L^{2}\left(\Omega^{\mathrm{e}}\right) \times L^{2}\left(\Omega^{\mathrm{a}}\right)\right)$, and $(\ddot{\boldsymbol{u}}, \ddot{\psi}) \in L^{2}\left(0, T ; H^{-1}\left(\Omega^{\mathrm{e}}\right) \times\right.$ $\left.H^{-1}\left(\Omega^{\mathrm{a}}\right)\right)$, such that for all times $t \in(0, T)$ :

$$
\begin{aligned}
&\left\langle\rho_{\mathrm{e}} \ddot{\boldsymbol{u}}, \boldsymbol{v}\right\rangle_{\Omega^{\mathrm{e}}}+a_{\mathrm{e}}(\boldsymbol{u}, \boldsymbol{v})+\langle\rho_{\mathrm{a}} \boldsymbol{v} \cdot \boldsymbol{n}, \overbrace{\gamma \psi}^{\dot{\gamma}}\rangle_{\Gamma}=l_{\mathrm{e}}(\boldsymbol{v}), \boldsymbol{v} \in X^{\mathrm{e}}, \\
&\left\langle\frac{\rho_{\mathrm{a}}}{c^{2}} \ddot{\psi}, w\right\rangle_{\Omega^{\mathrm{a}}}+a_{\mathrm{a}}^{\operatorname{lin}}\left(\psi, \rho_{\mathrm{a}} w\right)-\langle\rho_{\mathrm{a}} w, \overbrace{\gamma \boldsymbol{u} \cdot \boldsymbol{n}}\rangle_{\Gamma}=0, \quad w \in X^{\mathrm{a}} .
\end{aligned}
$$

Linear elastic/nonlinear acoustic continuous system

Find $(\boldsymbol{u}, \psi) \in L^{2}\left(0, T ; X^{\mathrm{e}} \times X^{\mathrm{a}}\right),(\dot{\boldsymbol{u}}, \dot{\psi}) \in L^{2}\left(0, T ; L^{2}\left(\Omega^{\mathrm{e}}\right) \times L^{2}\left(\Omega^{\mathrm{a}}\right)\right)$, and $(\ddot{\boldsymbol{u}}, \ddot{\psi}) \in L^{2}\left(0, T ; H^{-1}\left(\Omega^{\mathrm{e}}\right) \times\right.$ $\left.H^{-1}\left(\Omega^{\mathrm{a}}\right)\right)$, such that for all times $t \in(0, T)$ :

$$
\begin{aligned}
& \left\langle\rho_{\mathrm{e}} \ddot{\boldsymbol{u}}, \boldsymbol{v}\right\rangle_{\Omega^{\mathrm{e}}}+a_{\mathrm{e}}^{\operatorname{lin}}(\boldsymbol{u}, \boldsymbol{v})+\langle\rho_{\mathrm{a}} \boldsymbol{v} \cdot \boldsymbol{n}, \overbrace{\gamma \psi}^{\dot{\gamma}}\rangle_{\Gamma}=l_{\mathrm{e}}(\boldsymbol{v}), \quad \boldsymbol{v} \in X^{\mathrm{e}}, \\
& \left\langle\frac{\rho_{\mathrm{a}}}{c^{2}} \ddot{\psi}, w\right\rangle_{\Omega^{\mathrm{a}}}+a_{\mathrm{a}}\left(\psi, \rho_{\mathrm{a}} w\right)-\langle\rho_{\mathrm{a}} w, \overbrace{\boldsymbol{u} \cdot \boldsymbol{n}}^{\cdot}\rangle_{\Gamma}-\langle\rho_{\mathrm{a}} w, \frac{b}{c^{2}} \overbrace{\boldsymbol{u} \cdot \boldsymbol{n}}^{\ddot{n}}\rangle_{\Gamma}=0, \quad w \in X^{\mathrm{a}} .
\end{aligned}
$$

We remark that an alternative coupled problem can be derived, if, instead of the potential-based formulation, a displacement-based problem formulation is chosen also for the fluid domain. A rigorous mathematical error analysis for the purely linear case is provided in [2] for the case of matching grids which could be extended to the non-matching situation. There, due to the displacement-based formulation, the weak form of the subproblem for the fluid is an $H^{\text {div }}$-problem requiring a non-standard discretization by means of Raviart-Thomas finite elements. Here, we are able to use standard Lagrangian nodal finite elements in both domains.

\subsection{Discretization}

For the following considerations, the notation sets up on [12], in combination with $[1,5]$. For the time integration, we use the classical Newmark scheme $[10,14]$. The proposed algorithm is easily extendable to more sophisticated time stepping schemes like the generalized $\alpha$-method, but for the sake of brevity and clarity, we stick with this simple approach. In particular, we decompose the interval $[0, T]$ into subintervals $\left[t_{n}, t_{n+1}\right]$, $n=0, \ldots, N_{t}-1$, with $t_{n}:=n \Delta t, \Delta t:=T / N_{t}$. For a time-dependent quantity $x$, we denote by $x_{n} \approx$ $x(n \Delta t)$ its approximation at $t=t_{n}$. The defining feature of the classical Newmark method is to compute the approximations $\dot{x}_{n+1}$ and $\ddot{x}_{n+1}$ as functions of $x_{n+1}$ and the already known values $x_{n}, \dot{x}_{n}, \ddot{x}_{n}$,

$$
\begin{aligned}
& \dot{x}_{n+1}\left(x_{n+1}\right):=2 \Delta t^{-1}\left(x_{n+1}-x_{n}\right)-\dot{x}_{n}, \\
& \ddot{x}_{n+1}\left(x_{n+1}\right):=4 \Delta t^{-2}\left(x_{n+1}-x_{n}\right)-4 \Delta t^{-1} \dot{x}_{n}-\ddot{x}_{n} .
\end{aligned}
$$

It is well known that the classical Newmark scheme is unconditionally stable and of quadratic order with respect to time. 


\subsubsection{Structural part}

For the uncoupled structural model (2.4) on the spatially discrete level, this yields the system

$$
M_{\mathrm{e}} \ddot{\boldsymbol{u}}_{n+1}+N_{\mathrm{e}}\left(\boldsymbol{u}_{n+1}\right)=\boldsymbol{f}_{n+1}, \quad n \geq 0,
$$

with the constant mass matrix $M_{\mathrm{e}}$, the internal forces $N_{\mathrm{e}}$, and the external load $\boldsymbol{f}$, given in the standard way by $\left(\rho_{\mathrm{e}} \cdot, \cdot\right)_{\Omega^{\mathrm{e}}}, a_{\mathrm{e}}(\boldsymbol{u}, \cdot)$, and $l_{\mathrm{e}}(\cdot)$, respectively. We remark that for $n=-1$, the balance equation $(3.11)$ admits to calculate an initial acceleration $\ddot{\boldsymbol{u}}_{0}$ out of the knowledge of the initial values $\boldsymbol{u}_{0}$ and $\dot{\boldsymbol{u}}_{0}$ for displacement and velocity, respectively. Then, for $n \geq 0$, relations (3.10) can be employed to express $\ddot{\boldsymbol{u}}_{n+1}$ in terms of the unknown displacements $\boldsymbol{u}_{n+1}$ and known values from the last timestep. After new displacements $\boldsymbol{u}_{n+1}$ are found by means of (3.11), new velocities and accelerations are calculated via (3.10).

This gives the effective structural equation

$$
\mathbf{0}=\widetilde{F}_{\mathrm{e}}\left(\boldsymbol{u}_{n+1}\right):=M_{\mathrm{e}} \ddot{\boldsymbol{u}}_{n+1}\left(\boldsymbol{u}_{n+1}\right)+N_{\mathrm{e}}\left(\boldsymbol{u}_{n+1}\right)-\boldsymbol{f}_{n+1},
$$

where the dependence of $\ddot{\boldsymbol{u}}_{n+1}$ on $\boldsymbol{u}_{n+1}$ has to be seen with respect to (3.10). If we only consider a linear elasticity model corresponding to Section 2.2, the system above simplifies to

$$
\mathbf{0}=\widetilde{F}_{\mathrm{e}, \operatorname{lin}}\left(\boldsymbol{u}_{n+1}\right):=M_{\mathrm{e}} \ddot{\boldsymbol{u}}_{n+1}\left(\boldsymbol{u}_{n+1}\right)+K_{\mathrm{e}} \boldsymbol{u}_{n+1}-\boldsymbol{f}_{n+1},
$$

where the constant matrix $K_{\mathrm{e}}$ is associated with the linear part $a_{\mathrm{e}}^{\text {lin }}(\cdot, \cdot)$ of the elasticity bilinear form.

\subsubsection{Acoustic part}

The Newmark method applied to the weak form (2.7) of the wave equation (2.6a) for the acoustic velocity potential $\psi$ gives

$$
M_{\mathrm{a}} \ddot{\psi}_{n+1}+C_{\mathrm{a}} \dot{\psi}_{n+1}+K_{\mathrm{a}} \psi_{n+1}=N_{1}\left(\dot{\psi}_{n+1}\right) \ddot{\psi}_{n+1}+N_{2}\left(\psi_{n+1}\right) \dot{\psi}_{n+1},
$$

with constant matrices $M_{\mathrm{a}}, C_{\mathrm{a}}$, and $K_{\mathrm{a}}$ assembled from $\left(\frac{1}{c^{2}} \cdot, \cdot\right)_{\Omega^{\mathrm{a}}},\left(\frac{b}{c^{2}} \operatorname{grad} \cdot, \operatorname{grad} \cdot\right)_{\Omega^{\mathrm{a}}}, \operatorname{and}(\operatorname{grad} \cdot, \operatorname{grad} \cdot)_{\Omega^{\mathrm{a}}}$, respectively, as well as the nonlinear contributions given by

$$
\left(N_{1}(\dot{\psi})\right)_{i j}:=\int_{\Omega^{a}} \frac{B / A}{c^{4}} \phi^{i} \phi^{j} \dot{\psi}, \quad\left(N_{2}(\psi)\right)_{i j}:=\int_{\Omega^{a}} \frac{2}{c^{2}} \phi^{i} \operatorname{grad} \phi^{j} \cdot \operatorname{grad} \psi .
$$

Here, $\phi^{r}$ denotes the standard nodal basis functions for the acoustic potential associated with the node $r$. Similar as for the elastic part, we can write (3.14) in the form

$$
0=\widetilde{F}_{\mathrm{a}}\left(\psi_{n+1}\right):=M_{\mathrm{a}} \ddot{\psi}_{n+1}\left(\psi_{n+1}\right)+C_{\mathrm{a}} \dot{\psi}_{n+1}\left(\psi_{n+1}\right)+K_{\mathrm{a}} \psi_{n+1}-N_{\mathrm{a}}\left(\psi_{n+1}\right)
$$

where $N_{\mathrm{a}}\left(\psi_{n+1}\right)$ denotes the Newmark representation of the right hand side in (3.14). By simply dropping the nonlinear term $N_{\mathrm{a}}(\cdot)$, we obtain the fully discretized linear acoustic problem corresponding to Section 2.4,

$$
0=\widetilde{F}_{\mathrm{a}, \operatorname{lin}}\left(\psi_{n+1}\right):=M_{\mathrm{a}} \ddot{\psi}_{n+1}\left(\psi_{n+1}\right)+C_{\mathrm{a}} \dot{\psi}_{n+1}\left(\psi_{n+1}\right)+K_{\mathrm{a}} \psi_{n+1} .
$$

\subsubsection{Coupling and global systems}

It remains to realize the discrete coupling of the structural and the acoustic quantities. Corresponding to the boundary integrals appearing in $(3.7 \mathrm{~b})$, we define the coupling matrices $C_{\mathrm{ea}}$ and $M_{\mathrm{ae}}$ by

$$
\left(C_{\mathrm{ea}}\right)_{i j}:=\left(\rho_{\mathrm{a}} \boldsymbol{\phi}^{i} \cdot \boldsymbol{n}, \phi^{j}\right)_{\Gamma}, \quad\left(M_{\mathrm{ae}}\right)_{i j}:=-\left(\frac{\rho_{\mathrm{a}} b}{c^{2}} \boldsymbol{\phi}^{j} \cdot \boldsymbol{n}, \phi^{i}\right)_{\Gamma},
$$

where $\phi^{r}$ and $\phi^{s}$ denote basis functions associated with the elastic and the acoustic part, respectively. In particular, we remind that the surface grids inherited by the triangulations of the two subdomains do not need 
to match on $\Gamma$ in order to realize the coupling. Moreover, the matrices $C_{\mathrm{ea}}$ and $M_{\mathrm{ae}}$ do not depend on the solution.

In order to establish the connection to the abstract framework presented in Section 1, we set $x_{n+1}:=$ $\left(\boldsymbol{u}_{n+1}, \psi_{n+1}\right)^{\mathrm{T}}$ for the global solution to be determined in each time step. The fully discrete and fully nonlinear global system is now given by

$$
0=F\left(x_{n+1}\right):=\left(\begin{array}{l}
F_{\mathrm{e}}\left(x_{n+1}\right) \\
F_{\mathrm{a}}\left(x_{n+1}\right)
\end{array}\right):=\left(\begin{array}{c}
\widetilde{F}_{\mathrm{e}}\left(\boldsymbol{u}_{n+1}\right)+C_{\mathrm{ea}} \dot{\psi}_{n+1}\left(\psi_{n+1}\right) \\
\widetilde{F}_{\mathrm{a}}\left(\psi_{n+1}\right)-C_{\mathrm{ea}}^{\mathrm{T}} \dot{\boldsymbol{u}}_{n+1}\left(\boldsymbol{u}_{n+1}\right)+M_{\mathrm{ae}} \ddot{\boldsymbol{u}}_{n+1}\left(\boldsymbol{u}_{n+1}\right)
\end{array}\right) .
$$

As mentioned above, the coupling of a linear model in one part with a nonlinear model in the other part is of our particular interest. This amounts to replacing in (3.19) the nonlinear functions $\widetilde{F}_{i}\left(\boldsymbol{u}_{n+1}\right)$ defined by (3.12), (3.16) by the linear functions $\widetilde{F}_{i, \text { lin }}\left(\boldsymbol{u}_{n+1}\right)$ defined by (3.13), (3.17), respectively. In particular, we will elaborate on the following two systems.

Nonlinear elastic/linear acoustic discrete system

The global discrete system coupling nonlinear structural dynamics with linear acoustics and discretizing (3.8) reads

$$
0=F\left(x_{n+1}\right):=\left(\begin{array}{c}
F_{\mathrm{e}}\left(x_{n+1}\right) \\
F_{\mathrm{a}, \operatorname{lin}}\left(x_{n+1}\right)
\end{array}\right):=\left(\begin{array}{c}
\widetilde{F}_{\mathrm{e}}\left(\boldsymbol{u}_{n+1}\right)+C_{\mathrm{ea}} \dot{\psi}_{n+1}\left(\psi_{n+1}\right) \\
\left.\widetilde{F}_{\mathrm{a}, \operatorname{lin}}\left(\psi_{n+1}\right)-C_{\mathrm{ea}}^{\mathrm{T}} \dot{\boldsymbol{u}}_{n+1}\left(\boldsymbol{u}_{n+1}\right)\right)
\end{array}\right),
$$

where the mass coupling matrix $M_{\mathrm{ae}}$ vanishes since the corresponding boundary integral vanishes in (3.8b).

\section{Linear elastic/nonlinear acoustic discrete system}

The global discrete system coupling linear structural dynamics with nonlinear acoustics and discretizing (3.9) reads

$$
0=F\left(x_{n+1}\right):=\left(\begin{array}{c}
F_{\mathrm{e}, \operatorname{lin}}\left(x_{n+1}\right) \\
F_{\mathrm{a}}\left(x_{n+1}\right)
\end{array}\right):=\left(\begin{array}{c}
\widetilde{F}_{\mathrm{e}, \operatorname{lin}}\left(\boldsymbol{u}_{n+1}\right)+C_{\mathrm{ea}} \dot{\psi}_{n+1}\left(\psi_{n+1}\right) \\
\widetilde{F}_{\mathrm{a}}\left(\psi_{n+1}\right)-C_{\mathrm{ea}}^{\mathrm{T}} \dot{\boldsymbol{u}}_{n+1}\left(\boldsymbol{u}_{n+1}\right)+M_{\mathrm{ae}} \ddot{\boldsymbol{u}}_{n+1}\left(\boldsymbol{u}_{n+1}\right)
\end{array}\right) .
$$

\subsection{Analysis within the Schwarz framework}

Above, we have derived the requested form (1.1) of different nonlinearities in structure-acoustic interaction application. It remains to show that the theory of Section 1 can be applied to the systems (3.20) and (3.21). In particular, we have to verify Assumptions (A1)-(A3) from Theorem 1.1 by investigating the Jacobian $J(x)$, which in the case of the fully nonlinear problem (3.19) decomposes to

$$
J(x)=\left(\begin{array}{cc}
K_{\mathrm{e}}^{*}(\boldsymbol{u}) & \widetilde{B}_{\mathrm{ea}} \\
\widetilde{B}_{\mathrm{ae}} & K_{\mathrm{a}}^{*}(\psi)
\end{array}\right),
$$

with the effective elastic and acoustic stiffness matrices $K_{\mathrm{e}}^{*}(\boldsymbol{u})$ and $K_{\mathrm{a}}^{*}(\psi)$ given by

$$
K_{\mathrm{e}}^{*}(\boldsymbol{u})=\partial \widetilde{F}_{\mathrm{e}}(\boldsymbol{u}) / \partial \boldsymbol{u}, \quad K_{\mathrm{a}}^{*}(\psi)=\partial \widetilde{F}_{\mathrm{a}}(\psi) / \partial \psi,
$$

respectively, and the constant matrices $B_{\mathrm{ea}}=2 \Delta t^{-1} C_{\mathrm{ea}}$ and $B_{\mathrm{ae}}=-B_{\mathrm{ea}}^{\mathrm{T}}+4 \Delta t^{-2} M_{\mathrm{ae}}$, due to (3.19) in combination with (3.10). Above and in the sequel, the subscript $n+1$ is dropped for convenience. We will separately treat problems (3.20) and (3.21) by means of the following two lemmas. 
Lemma 3.1. Assume that $\Delta t$ is small enough. Then, Assumptions (A1)-(A3) hold for F defined by (3.20) and the decomposition introduced above.

Proof. By the definition of $F$ in (3.20), the Jacobian $J(x)$ simplifies from the fully nonlinear case (3.22) to

$$
J(x)=\left(\begin{array}{cc}
K_{\mathrm{e}}^{*}(\boldsymbol{u}) & B^{\mathrm{T}} \\
-B & K_{\mathrm{a}}^{*}
\end{array}\right),
$$

with $K_{\mathrm{a}}^{*}$ defined according to (3.17) and (3.10) by

$$
K_{\mathrm{a}}^{*}:=K_{\mathrm{a}}+2 \Delta t^{-1} C_{\mathrm{a}}+4 \Delta t^{-2} M_{\mathrm{a}},
$$

and $B^{\mathrm{T}}:=2 \Delta t^{-1} C_{\text {ea }}$. Being constant, it is obvious that $K_{\mathrm{a}}^{*}$ and $B$ are continuous. Moreover, due to the use of the geometrically nonlinear elasticity model (2.1)-(2.3a), the effective tangent matrix $K_{\mathrm{e}}^{*}(\boldsymbol{u})$ is given in terms of polynomials of the gradient $\operatorname{grad} \boldsymbol{u}$, and is also continuous, which proves (A1). The subdomain matrices $E_{i} J\left(x^{*}\right) E_{i}^{\mathrm{T}}, i=\mathrm{e}$, a, are given by $K_{\mathrm{e}}^{*}(\boldsymbol{u})$ and $K_{\mathrm{a}}^{*}$, respectively. Looking closer at $K_{\mathrm{e}}^{*}(\boldsymbol{u})$ by means of $(3.23),(3.12)$, and $(3.10)$, we see that

$$
K_{\mathrm{e}}^{*}(\boldsymbol{u})=\partial N_{\mathrm{e}}(\boldsymbol{u}) / \partial \boldsymbol{u}+4 \Delta t^{-2} M_{\mathrm{e}} .
$$

Therefore, one component of both stiffness matrices is the multiple $4 \Delta t^{-2} M_{i}$ with $M_{i}$ being the corresponding mass matrix, and thus both matrices are positive definite for $\Delta t$ small enough, regardless of the underlying boundary conditions. This shows (A3), and (A2) follows immediately.

Lemma 3.2. Assume that $b$ and $c$ are constant, and that $\Delta t$ is small enough. Then, Assumptions (A1)-(A3) hold for $F$ defined by (3.21) and the decomposition introduced above.

Proof. We can alter the proof from Lemma 3.1 in order to use it for the linear elastic/nonlinear acoustic problem (3.21). Now, the Jacobian $J(x)$ simplifies from (3.22) to

$$
J(x)=\left(\begin{array}{cc}
K_{\mathrm{e}}^{*} & B_{\mathrm{ea}} \\
B_{\mathrm{ae}} & K_{\mathrm{a}}^{*}(\psi)
\end{array}\right),
$$

with $K_{\mathrm{e}}^{*}$ defined according to (3.13) and (3.10) by

$$
K_{\mathrm{e}}^{*}:=K_{\mathrm{e}}+4 \Delta t^{-2} M_{\mathrm{e}}
$$

From definition (3.15), we see that $K_{\mathrm{a}}^{*}(\psi)$ depends continuously on $\psi$ and $\operatorname{grad} \psi$. Together with the fact that $K_{\mathrm{e}}^{*}$, $B_{\text {ea }}$, and $B_{\text {ae }}$ are constant, condition (A1) is fulfilled. Furthermore, we set as before $B^{T}:=B_{\text {ea }}:=2 \Delta t^{-1} C_{\text {ea }}$. Since $b$ and $c$ are assumed to be constant, we have $M_{\mathrm{ae}}=-b c^{-2} C_{\mathrm{ea}}^{T}$ due to (3.18), and hence $B_{\mathrm{ae}}=a B$ with $a=-\left(1+2 \Delta t^{-1} b c^{-2}\right)$. Thus, we have that

$$
J(x)=\left(\begin{array}{cc}
K_{\mathrm{e}}^{*} & B^{T} \\
a B & K_{\mathrm{a}}^{*}(\psi)
\end{array}\right) .
$$

We can simply divide the last line and the corresponding right hand side by $a$, yielding

$$
J(x):=\left(\begin{array}{cc}
K_{\mathrm{e}}^{*} & B^{T} \\
B & a^{-1} K_{\mathrm{a}}^{*}(\psi)
\end{array}\right)
$$

for which similar arguments as for (3.24) hold. 


\subsection{The iterative solution procedures}

We present the overall iterative solution procedure for system (3.20) in Algorithm 1 and for system (3.21) in Algorithm 2. The essential algorithmic difference is that we use Newton's method for the resolution of the nonlinear elastic subproblem, while we employ a fixed point iteration for the nonlinear acoustic part.

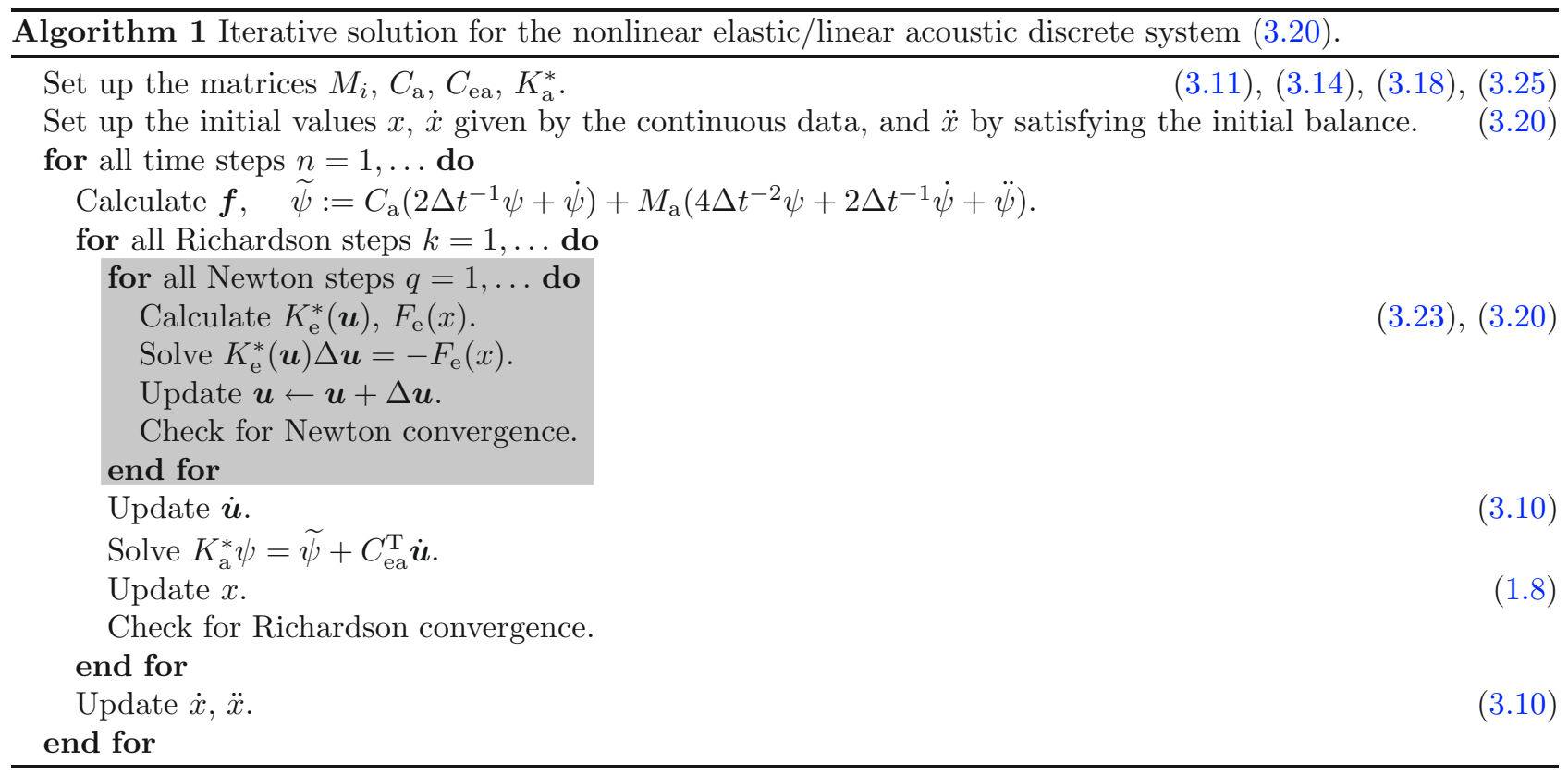

For system (3.20) we remark that most of the matrices only have to be assembled once in the very beginning, and only the relatively small tangential stiffness matrix $K_{\mathrm{e}}^{*}(\boldsymbol{u})$ has to be calculated in every Newton step. The only difference between the additive and the multiplicative variant is the update of the displacement degrees of freedom before the solution for the new velocity potential in the latter case. Thus, as in the linear case, for single processor architectures, the costs per iteration step are almost the same, and the multiplicative variant is clearly superior to the additive one. As is well-known, this argument has to be reconsidered in the case of parallel computing architectures.

\section{NumericAl COMPUTATIONS}

We provide several numerical results illustrating the applicability and efficiency of our approach. In particular, we validate our theoretical results in Section 4.1, and discuss some further improvements in Sections 4.2 and 4.3.

\subsection{Validation of the theoretical results}

In the following, we investigate Theorems 1.2 and 1.7 by means of a numerical example simulating the sound emission of a vibrating structure in water. The structure $\Omega^{\mathrm{e}}$ is of width $0.2 \mathrm{~m}$ and height $0.01 \mathrm{~m}$ and has the material parameters of silicon, $\rho_{\mathrm{e}}:=2.3 \times 10^{3} \mathrm{~kg} \cdot \mathrm{m}^{-3}, E:=1.62 \times 10^{11} \mathrm{~N} \cdot \mathrm{m}^{-2}, \nu:=0.2$. It is fixed at its left and right boundary, and a surface traction of $10^{8} \sin (2 \pi 1000 t) \mathrm{N} \cdot \mathrm{m}^{-2}$ is acting in vertical direction at its lower boundary. We assume the structure to behave geometrically nonlinear. The acoustic fluid domain $\Omega^{\mathrm{a}}$ of width $1.4 \mathrm{~m}$ and height $0.7 \mathrm{~m}$ is centered above the structure. The mean density is $\rho_{\mathrm{a}}:=10^{3} \mathrm{~kg} \cdot \mathrm{m}^{-3}$, the speed of sound is $c:=1.5 \times 10^{3} \mathrm{~m} \cdot \mathrm{s}^{-1}$. The corresponding parameters for the nonlinear wave equation in water are $B / A:=5$ and $b:=1.83 \times 10^{-10} \mathrm{~m}^{2} \cdot \mathrm{s}^{-1}$. Due to symmetry reasons, we can set the computational domain to one half of the original one, see Figure 2. As described in [8], we are able to use nonmatching grids in the different subdomains. This allows us to be as flexible as possible in the choice of the grids. 
(a)

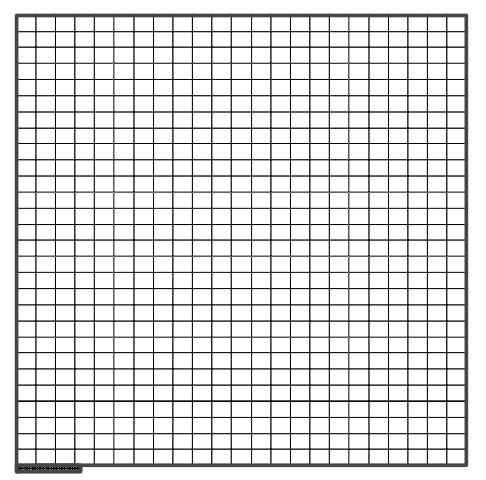

(b)

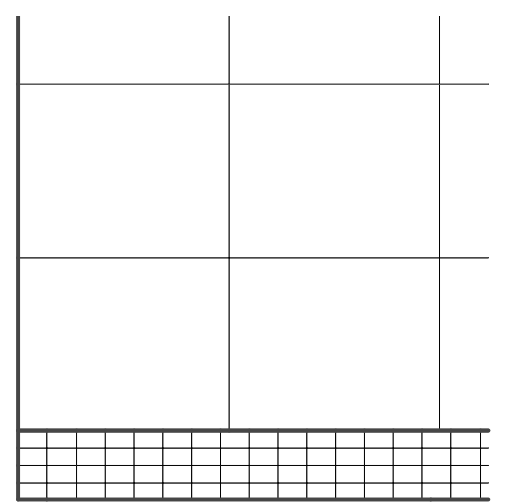

Figure 2. (a) Computational grid: lower small part $=$ structure, upper part $=$ acoustic; (b) use of nonmatching grids.

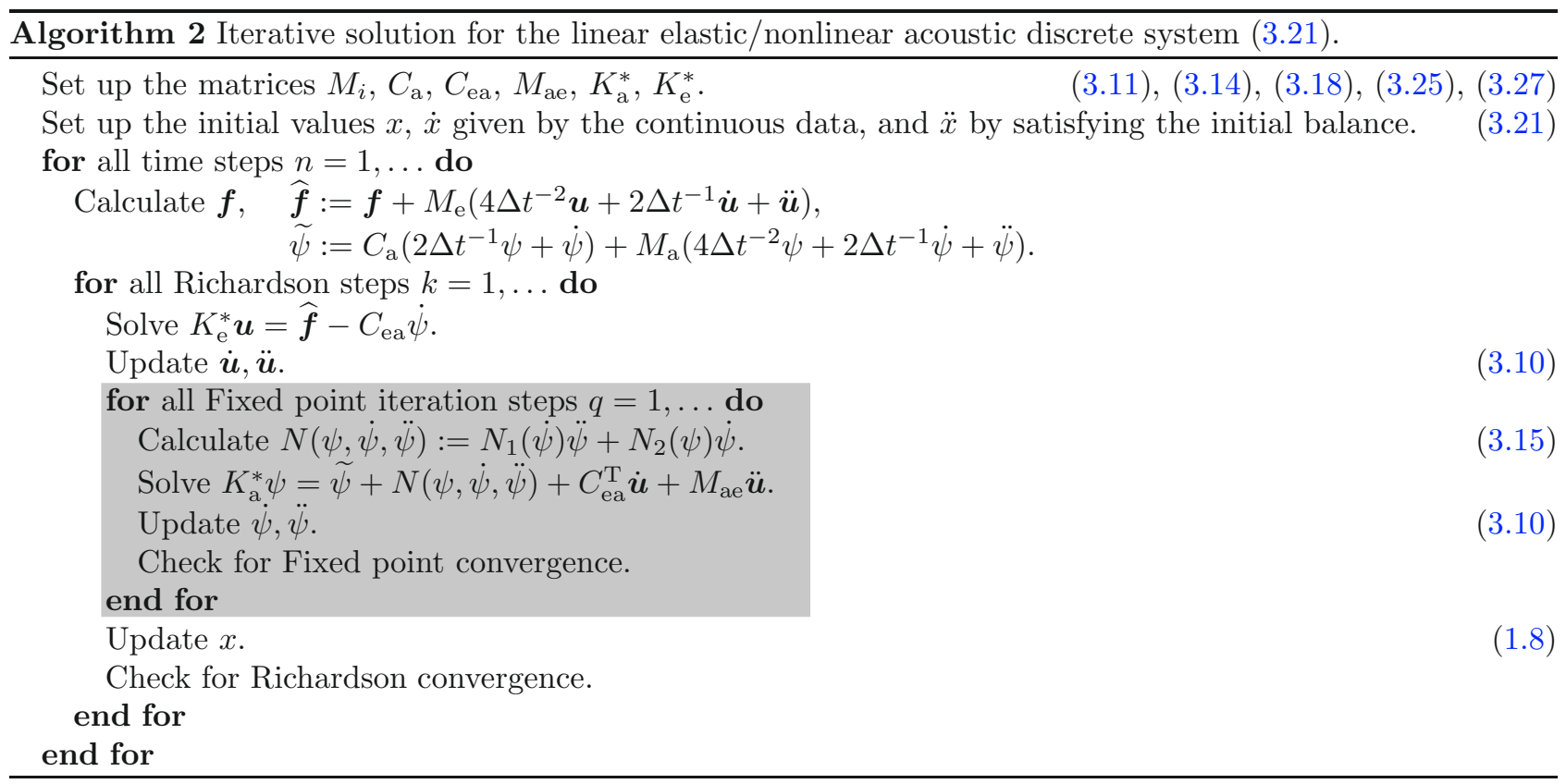

A test series with varying damping parameter $\omega$ is performed. In each test, 20 time steps of size $2.5 \times 10^{-5} \mathrm{~s}$ are carried out. Figures $3 \mathrm{a}$ and $3 \mathrm{~b}$ show the averaged convergence rate versus the value of $\omega$ for the nonlinear scheme (1.4) and for its linearized variant. We observe that even the averaged convergence rate of the nonlinear iteration, which is directly related to the number of iterations necessary to achieve a desired reduction of the error, is in good agreement with the one of the linear iteration. This nicely confirms and even strengthens the statement of Theorem 1.2. Moreover, for this particular example, a damping parameter $\omega:=0.7$ seems to be optimal. However, the minimum achievable convergence rate of around 0.55 is far too high for a block $2 \times 2$-scheme to be efficient. In the following section, we therefore will gradually improve our iteration scheme.

We perform the same test series for the multiplicative variant (1.8) as described above. In Figure 4, the resulting averaged convergence rates are plotted against the damping parameter as before. Additionally, the result for the additive variant from before is given for comparison. 

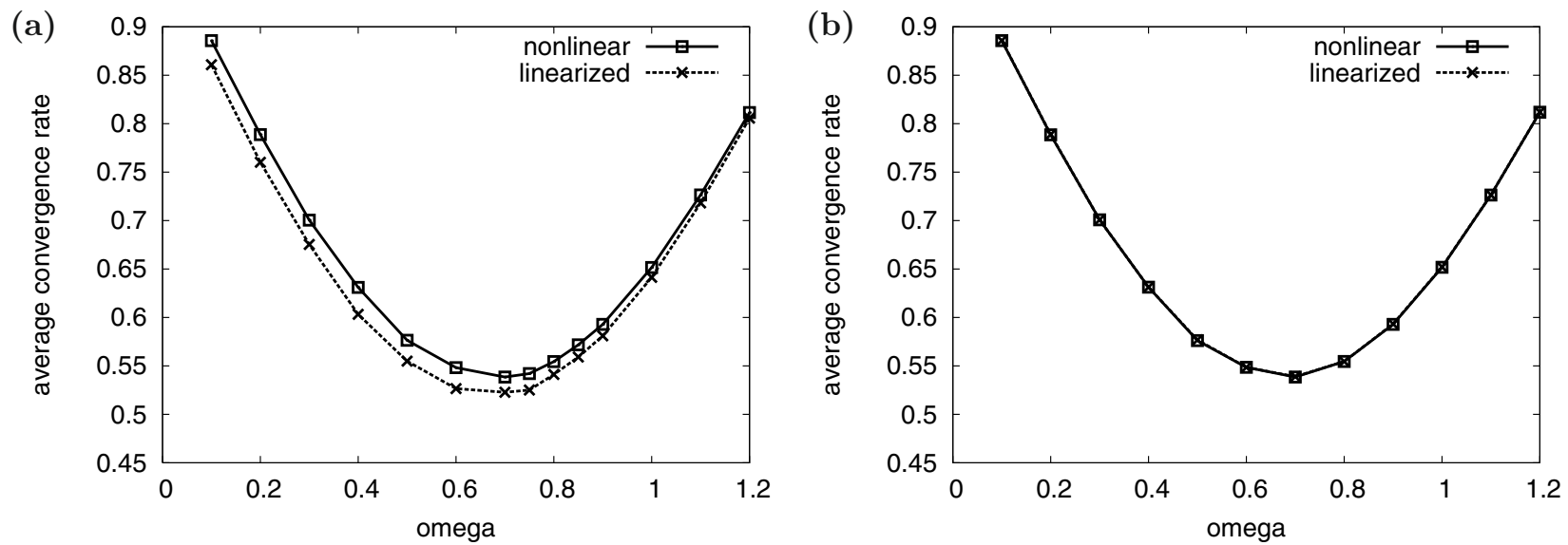

Figure 3. Test of the additive Richardson scheme (1.4): average convergence rate versus damping parameter (a) for system (3.20) and (b) for system (3.21).
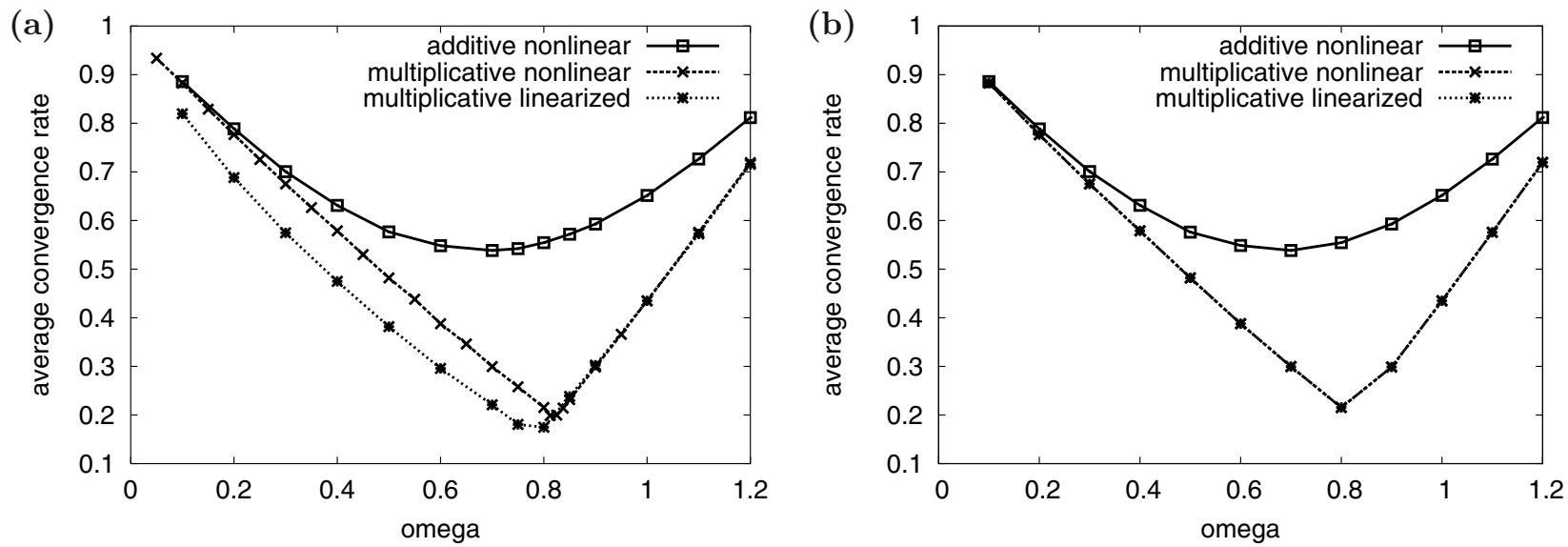

FIgURE 4. Test of the multiplicative variant (1.8): convergence rate versus damping parameter (a) for system (3.20) and (b) for system (3.21).

We observe that the nonlinear and the linearized multiplicative variant are in reasonable agreement. However, for the system (3.20), the difference to the left of the optimum value becomes quite remarkable. More important, the convergence rates are in general much better than for the additive Jacobi scheme. It is possible to reach a minimum value of roughly 0.2 . Yet, this is still quite high for a block $2 \times 2$-system. We will discuss a further improvement in the next section.

\subsection{Overlapping subdomains}

In order to improve the convergence behavior of the Richardson scheme, we further decompose $\psi$ into $\left(\psi_{1}, \psi_{2}, \lambda\right)$, where $\boldsymbol{u}$ couples with $\psi_{1}$ via $C_{\text {ea }}$, and $\psi_{1}$ with $\psi_{2}$ via the Lagrange multiplier $\lambda$ in the manner of mortar finite element methods [3,4]. We note that this procedure admits to use nonconforming grids in the acoustic subdomains associated with $\psi_{1}$ and $\psi_{2}$. However, we will not exploit this fact in our presentation.

In Figure 5, the computational grid is plotted, visualizing the structure in the lower left part. The thicker horizontal line inside the acoustic domain indicates the upper boundary of the overlapping region. We first concentrate on system (3.20). For the effective stiffness matrix associated with $\Omega^{\mathrm{a}}$ and arranged with respect 


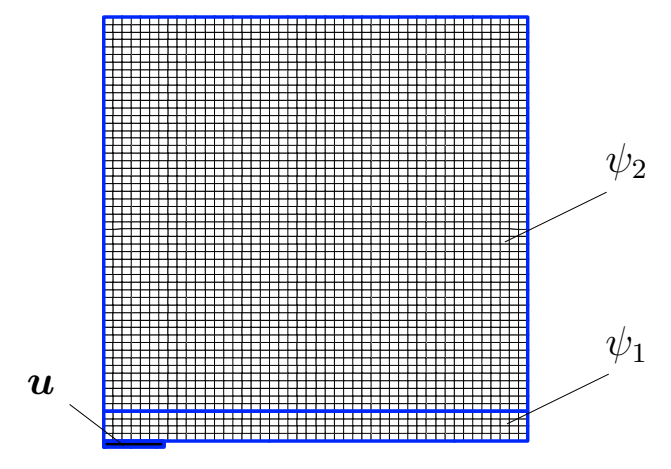

Figure 5. Computational domain with four layers overlap.

to the decomposition into $\left(\psi_{1}, \psi_{2}, \lambda\right)$, we have

$$
K_{\mathrm{a}}^{*}=\left(\begin{array}{ccc}
K_{11} & 0 & K_{1 \lambda} \\
0 & K_{22} & K_{2 \lambda} \\
K_{\lambda 1} & K_{\lambda 2} & 0
\end{array}\right)+a_{1}\left(\begin{array}{ccc}
C_{1} & 0 & 0 \\
0 & C_{2} & 0 \\
0 & 0 & 0
\end{array}\right)+a_{2}\left(\begin{array}{ccc}
M_{1} & 0 & 0 \\
0 & M_{2} & 0 \\
0 & 0 & 0
\end{array}\right)
$$

with the obvious meanings for the submatrices. We now choose a decomposition of $(1, \ldots, m)$ into $I_{\mathrm{e}}^{\mathrm{o}}$ and $I_{\mathrm{a}}$ with $I_{\mathrm{a}}$ as before including the indices associated with the Lagrange multiplier $\lambda$, but with $I_{\mathrm{e}}^{\mathrm{o}}$ being $I_{\mathrm{e}}$ enhanced by the indices associated with $\psi_{1}$. Now, this decomposition is overlapping, i.e., $\left|I_{\mathrm{e}}\right|+\left|I_{\mathrm{a}}\right|>m$. The resulting subspace function $F_{\mathrm{e}}^{\mathrm{o}}$ associated with $I_{\mathrm{e}}^{\mathrm{o}}$ is given by

$$
F_{\mathrm{e}}^{\mathrm{o}}(x):=P_{\mathrm{e}}^{\mathrm{o}} F(x)=\left(\begin{array}{c}
F_{\mathrm{e}}(x) \\
\left(F_{\mathrm{a}, \operatorname{lin}}(x)\right)_{1}+K_{1 \lambda} \lambda
\end{array}\right)
$$

where $\left(F_{\mathrm{a}, \text { lin }}(x)\right)_{1}$ is defined analogously to (3.20), but only contains the components associated with $\psi_{1}$. We now apply the Richardson iteration (1.8) to the preconditioned system

$$
0=\mathcal{F}_{\mathrm{m}}^{\mathrm{o}}\left(x^{*}\right):=T_{\mathrm{e}}^{\mathrm{o}}\left(x^{*}\right)+T_{\mathrm{a}}\left(x^{*}-T_{\mathrm{e}}^{\mathrm{o}}\left(x^{*}\right)\right) .
$$

For the resulting algorithm, only the Newton iteration has to be changed, i.e. the shaded region in Algorithm 1. The modified Newton scheme is given in Algorithm 3.

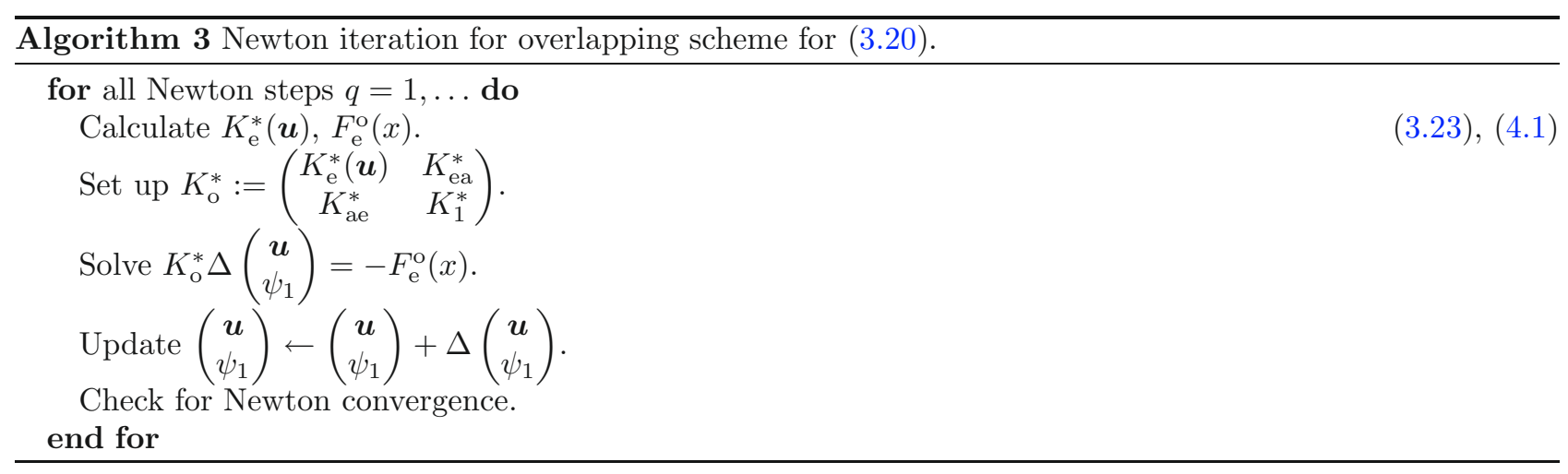


(a)

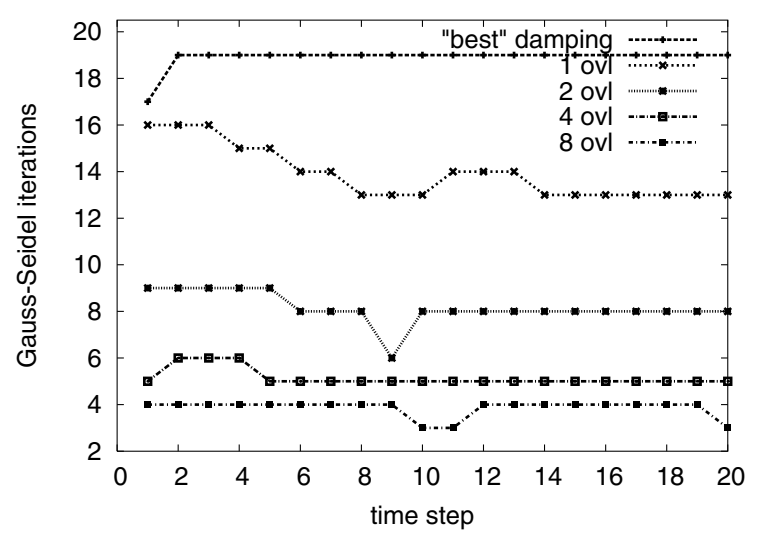

(b)

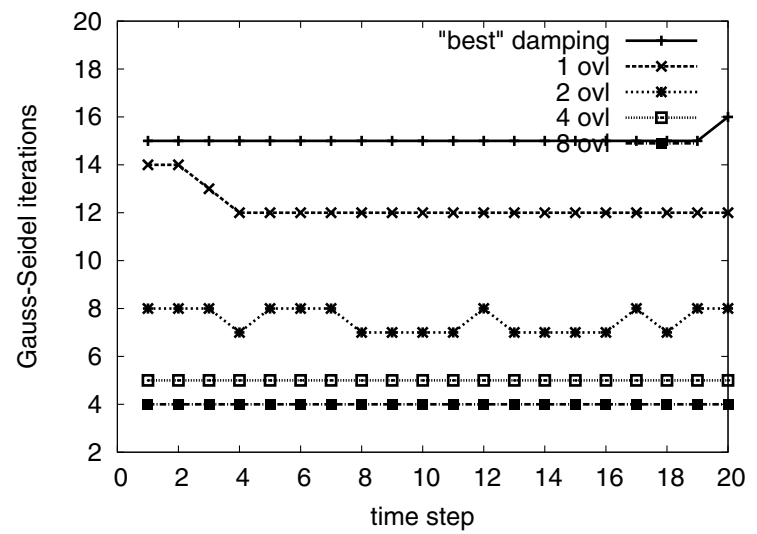

Figure 6 . Test of overlapping scheme: iteration count versus time step, varying the overlap (a) for system (3.20) and (b) for system (3.21).

The goal of this overlapping scheme is to include in $I_{\mathrm{e}}^{\mathrm{o}}$ only comparatively few degrees of freedom from the acoustic part, such that the computational costs for one iteration step are not much higher than for the non-overlapping variant, but to overlap enough to substantially improve the convergence rate.

In the case of the linear elastic/nonlinear acoustic system (3.21), we also introduce the overlap in the acoustic region. In contrast to Algorithm 3 we solve the linearized acoustic equation, i.e. we only make one fixed point iteration step, on the overlapping region. With the same notations as above we only have to change the equation outside the fixed point iteration in Algorithm 2 as is written in Algorithm 4.

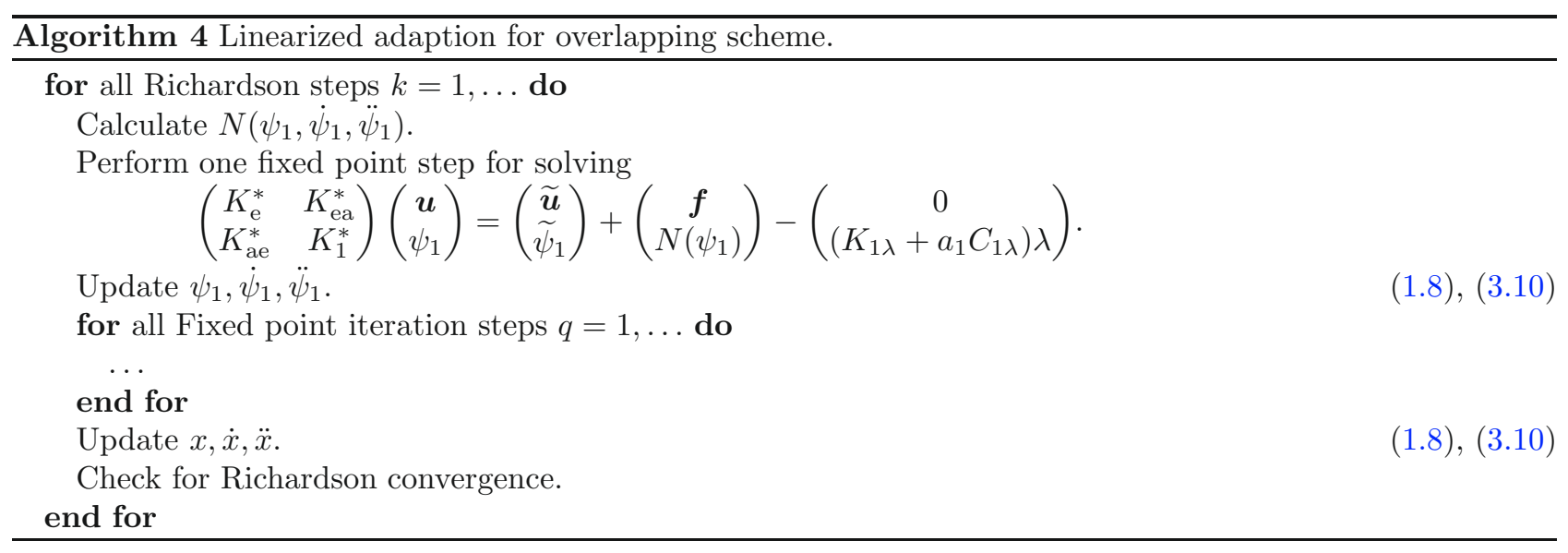

We use the same example as above to investigate the behaviour of the overlapping method. In particular, we vary the thickness of the overlap. In Figures $6 \mathrm{a}$ and $6 \mathrm{~b}$, the iteration counts for different numbers of overlapping layers are visualized against the time steps. Convergence for the Richardson iteration was declared, when the relative difference of two successive iterates went below $10^{-10}$. Already a very small number of layers results in a strong improvement. With four or eight layers, the count is around 5 or 4 , which is quite reasonable.

\subsection{An inexact strategy}

For the system (3.8), so far, in each Richardson step, there have been made so many Newton steps as necessary to meet the stopping criteria of the relative difference of two iterates to be below $10^{-10}$. In Figure 7 , 


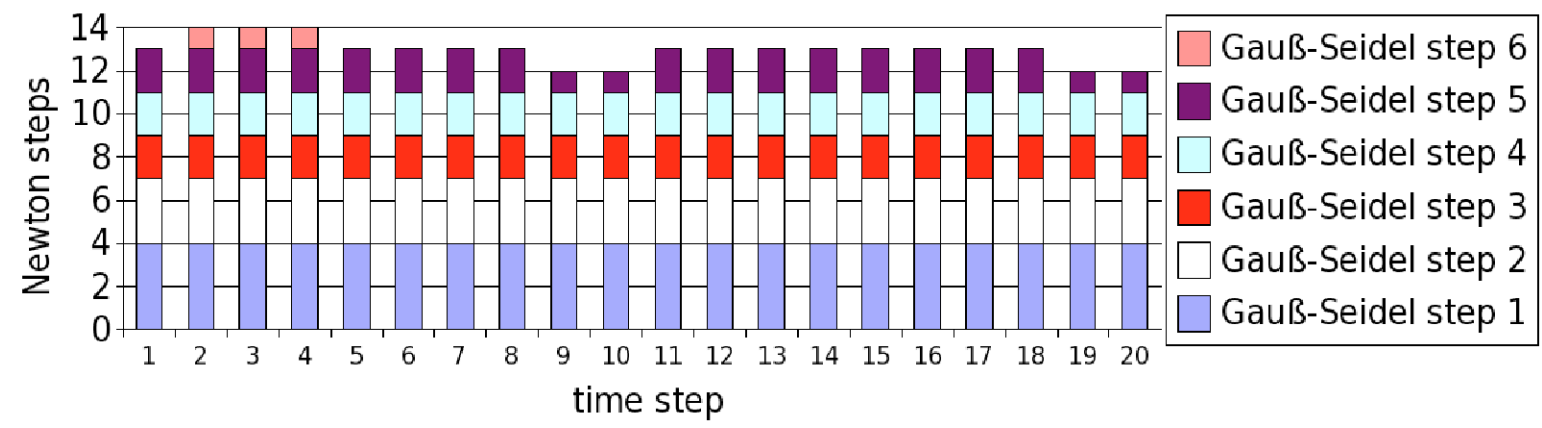

FIGURE 7. Overlapping multiplicative scheme, four layers of overlap: Newton iterations versus time step.
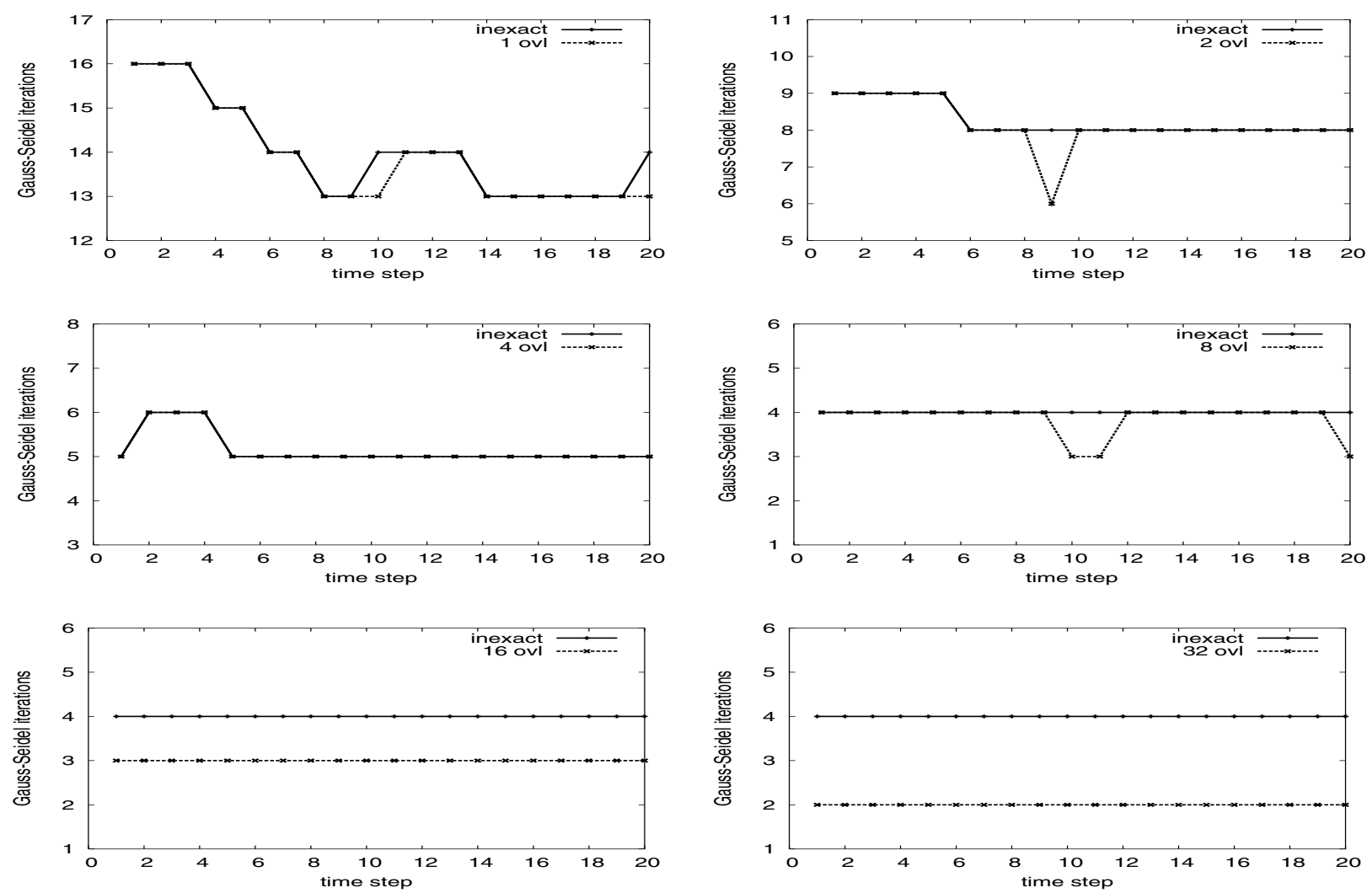

Figure 8. Overlapping multiplicative scheme: inexact (solid line) versus exact (dashed) strategy.

the number of Newton iterations is plotted versus the time step for the multiplicative scheme using four layers of overlap. In particular, the total number of Newton iterations per time step determines the height of the columns, whereas each column is subdivided to display the number of Newton iterations per Richardson step within each time step. The first observation to be made is that the total number of Newton iterations per time step is almost constant, as already suggested by the stable behaviour of the multiplicative scheme. Moreover, the number of Newton iterations in each Richardson step is very low. Starting from the solution of the previous time step, it only takes four Newton iterations in the first Richardson step, three in the second, and between 
one and two in the subsequent steps. Nevertheless, the efficiency of the scheme can further be improved by employing an inexact strategy.

To this end, only one single Newton step is performed in each Richardson step. Figure 8 shows the resulting iteration count for the multiplicative scheme versus the time steps for different numbers of overlapping layers. Up to eight layers, almost no difference between the exact and the inexact method can be observed. This means that the number of Newton steps per time step reduces significantly to the number of Richardson steps per time step. We remark that the resulting number of Newton steps per time step for the inexact strategy seems to be bounded from below by the number of Newton steps for the first Richardson step of the exact strategy. Thus, if the size of the overlap further increases, the iteration count for the inexact method becomes worse than the one for the exact method. However, in the reasonable range between four and eight layers of overlap, the inexact strategy is clearly superior.

Acknowledgements. The authors Bernd Flemisch and Barbara Wohlmuth express their gratitude to the German Academic Exchange Service DAAD for funding their stays at the University of Milano, where large parts of this paper have been created. They also wish to thank Luca Pavarino for several fruitful discussions during these stays.

\section{REFERENCES}

[1] H.-B. An, On convergence of the additive Schwarz preconditioned inexact Newton method. SIAM J. Numer. Anal. 43 (2005) $1850-1871$

[2] A. Bermúdez, R. Rodríguez and D. Santamarina, Finite element approximation of a displacement formulation for time-domain elastoacoustic vibrations. J. Comput. Appl. Math. 152 (2003) 17-34.

[3] C. Bernardi, Y. Maday and A.T. Patera, Domain decomposition by the mortar element method, in Asymptotic and numerical methods for partial differential equations with critical parameters (Beaune, 1992), NATO Adv. Sci. Inst. Ser. C Math. Phys. Sci. 384, Kluwer Acad. Publ., Dordrecht (1993) 269-286.

[4] C. Bernardi, Y. Maday and A.T. Patera, A new nonconforming approach to domain decomposition: the mortar element method, in Nonlinear partial differential equations and their applications, Collège de France Seminar, Vol. XI (Paris, 19891991), Pitman Res. Notes Math. Ser. 299, Longman Sci. Tech., Harlow (1994) 13-51.

[5] X.-C. Cai and D.E. Keyes, Nonlinearly preconditioned inexact Newton algorithms. SIAM J. Sci. Comput. 24 (2002) $183-200$.

[6] P.G. Ciarlet, Mathematical elasticity, Vol. I: Three-dimensional elasticity, Studies in Mathematics and its Applications 20. North-Holland Publishing Co., Amsterdam (1988).

[7] M. Dryja and W. Hackbusch, On the nonlinear domain decomposition method. BIT 37 (1997) 296-311.

[8] B. Flemisch, M. Kaltenbacher and B.I. Wohlmuth, Elasto-acoustic and acoustic-acoustic coupling on non-matching grids. Int. J. Numer. Meth. Engng. 67 (2006) 1791-1810.

[9] M.F. Hammilton and D.T. Blackstock, Nonlinear Acoustics. Academic Press (1998).

[10] T. Hughes, The Finite Element Method. Prentice-Hall, New Jersey (1987).

[11] M. Kaltenbacher. Numerical Simulation of Mechatronic Sensors and Actuators. Springer, Berlin-Heidelberg-New York (2007).

[12] D. Kuhl and M.A. Crisfield, Energy-conserving and decaying algorithms in non-linear structural dynamics. Int. J. Numer. Meth. Engng. 45 (1999) 569-599.

[13] V.I. Kuznetsov, Equations of nonlinear acoustics. Soviet Phys.-Acoust. 16 (1971) 467-470.

[14] N.M. Newmark, A method of computation for structural dynamics. J. Engng. Mech. Div., Proc. ASCE 85 (EM3) (1959) 67-94.

[15] A. Quarteroni and A. Valli, Domain decomposition methods for partial differential equations, Numerical Mathematics and Scientific Computation. Oxford University Press, New York (1999).

[16] A.-M. Sändig, Nichtlineare Funktionalanalysis mit Anwendungen auf partielle Differentialgleichungen. Vorlesung im Sommersemester 2006, IANS preprint 2006/012, Technical report, University of Stuttgart, Germany (2006).

[17] B.F. Smith, P.E. Bjørstad and W.D. Gropp, Domain decomposition, Parallel multilevel methods for elliptic partial differential equations. Cambridge University Press, Cambridge (1996).

[18] A. Toselli and O. Widlund, Domain decomposition methods - algorithms and theory, Springer Series in Computational Mathematics 34. Springer-Verlag, Berlin (2005). 